ARTICLE

\title{
Crystal structure of the $\alpha_{1 \mathrm{~B}}$-adrenergic receptor reveals molecular determinants of selective ligand recognition
}

\author{
Mattia Deluigi (1) 1, Lena Morstein (1) 1,10, Matthias Schuster 2,10, Christoph Klenk (D) 1, Lisa Merklinger ${ }^{1,7}$, \\ Riley R. Cridge ${ }^{3}$, Lazarus A. de Zhang ${ }^{3,4}$, Alexander Klipp (1) 1,8, Santiago Vacca (1) ${ }^{1}$, Tasneem M. Vaid ${ }^{5}$, \\ Peer R. E. Mittl (10 1, Pascal Egloff (1) ${ }^{1}$, Stefanie A. Eberle ${ }^{1,9}$, Oliver Zerbe (1) ${ }^{2}$, David K. Chalmers (1) ${ }^{6}$, \\ Daniel J. Scott ${ }^{3,4 凶} \&$ Andreas Plückthun (1) ${ }^{1 凶}$
}

$\alpha$-adrenergic receptors ( $\alpha A R s$ ) are $G$ protein-coupled receptors that regulate vital functions of the cardiovascular and nervous systems. The therapeutic potential of $\alpha A R s$, however, is largely unexploited and hampered by the scarcity of subtype-selective ligands. Moreover, several aminergic drugs either show off-target binding to $\alpha A R s$ or fail to interact with the desired subtype. Here, we report the crystal structure of human $\alpha_{1 B} A R$ bound to the inverse agonist (+)-cyclazosin, enabled by the fusion to a DARPin crystallization chaperone. The $\alpha_{1 B} A R$ structure allows the identification of two unique secondary binding pockets. By structural comparison of $\alpha_{1 B} A R$ with $\alpha_{2} A R s$, and by constructing $\alpha_{1 B} A R-\alpha_{2 C} A R$ chimeras, we identify residues 3.29 and 6.55 as key determinants of ligand selectivity. Our findings provide a basis for discovery of $\alpha_{1 B} A R$-selective ligands and may guide the optimization of aminergic drugs to prevent off-target binding to $\alpha$ ARs, or to elicit a selective interaction with the desired subtype.

\footnotetext{
${ }^{1}$ Department of Biochemistry, University of Zurich, Winterthurerstrasse 190, CH-8057 Zurich, Switzerland. ${ }^{2}$ Department of Chemistry, University of Zurich Winterthurerstrasse 190, CH-8057 Zurich, Switzerland. ${ }^{3}$ The Florey Institute of Neuroscience and Mental Health, The University of Melbourne, 30 Royal Parade, Parkville, VIC 3052, Australia. ${ }^{4}$ Department of Biochemistry and Pharmacology, The University of Melbourne, Parkville, VIC 3010, Australia. ${ }^{5}$ Department of Pharmaceutical Sciences, University of Illinois at Chicago, Chicago, IL, USA. ${ }^{6}$ Monash Institute of Pharmaceutical Sciences, Monash University, 381 Royal Parade, Parkville, VIC 3052, Australia. ${ }^{7}$ Present address: Department of Biotechnology and Biomedicine, Technical University of Denmark, Søltofts Plads, 2800 Kgs Lyngby, Denmark. ${ }^{8}$ Present address: Department of Chemistry and Applied Biosciences, ETH Zurich, Vladimir-PrelogWeg 1-5/10, CH-8093 Zurich, Switzerland. ${ }^{9}$ Present address: Department of Biomedical Sciences, Faculty of Health and Medical Sciences, University of Copenhagen, Blegdamsvej 3B, 2200 Copenhagen, Denmark. ${ }^{10}$ These authors contributed equally: Lena Morstein, Matthias Schuster.

凶email: daniel.scott@florey.edu.au; plueckthun@bioc.uzh.ch
} 
drenergic receptors (ARs), or adrenoceptors, are aminergic $\mathrm{G}$ protein-coupled receptors (GPCRs) subdivided into nine distinct subtypes in humans ${ }^{1,2}$-three $\alpha_{1}$ ARs $\left(\alpha_{1 A}\right.$, $\left.\alpha_{1 B}, \alpha_{1 D}\right)$, three $\alpha_{2}$ ARs $\left(\alpha_{2 A}, \alpha_{2 B}, \alpha_{2 C}\right)$, and three $\beta$ ARs $\left(\beta_{1}, \beta_{2}, \beta_{3}\right)$. ARs are widely yet differentially expressed in the central nervous system (CNS) and in peripheral sympathetic nerves, as well as in sympathetically innervated tissues throughout the body ${ }^{3,4}$. Upon activation by the endogenous catecholamines epinephrine and norepinephrine, ARs mediate a large variety of physiological functions, many of which are of considerable clinical relevance ${ }^{4}$. For instance, drugs blocking $\beta_{1} \mathrm{AR}$ are widely prescribed to treat hypertension and heart failure, while $\beta_{2} \mathrm{AR}$ agonists are used as bronchodilators in asthma therapy ${ }^{4,5}$.

In contrast to $\beta A R s$, the therapeutic potential of aARs is largely unexploited for at least two reasons. First, there is a lack of truly selective ligands for individual $\alpha_{1} A R$ and $\alpha_{2} A R$ subtypes, which often mediate opposing physiological functions ${ }^{6,7}$. As a consequence, drugs that act through aARs are only second-line agents to treat hypertension, pain, or neuropsychiatric disorders, due to their off-target side effects and limited therapeutic benefits ${ }^{6}$. Second, there is an incomplete understanding of the individual aAR subtypes' physiological and pathophysiological roles, as the lack of selective compounds has hampered research. Nonetheless, studies in transgenic mice indicate that distinct $\alpha_{1} \mathrm{AR}$ subtypes mediate different functions in the heart, CNS, and urogenital system ${ }^{7-9}$. Stimulation of $\alpha_{1 A} A R$ and $\alpha_{1 B} A R$, respectively, reduces or augments the severity of cardiac hypertrophy, heart failure, and ischemic disease. In the CNS, $\alpha_{1 \mathrm{~A}} \mathrm{AR}$ stimulation is antiepileptic and enhances neurogenesis, whereas excessive $\alpha_{1 B} A R$ activation is detrimental to brain function. In addition, $\alpha_{1}$ ARs contribute to the regulation of immune cells ${ }^{10,11}$, and pre-clinical studies suggest that $\alpha_{1}$ AR-blockers may protect against hyperinflammatory responses ${ }^{11-13}$. The $\alpha_{1} \mathrm{AR}$ antagonist prazosin is currently under evaluation in clinical trials for the prevention of cytokine storm syndrome caused by the severe acute respiratory syndrome coronavirus 2 (SARS-CoV-2), a leading cause of morbidity and mortality in coronavirus disease 2019 (COVID-19; https:// clinicaltrials.gov/ct2/show/NCT04365257) ${ }^{12}$. Similar to $\alpha_{1}$ ARs, individual $\alpha_{2} \mathrm{AR}$ subtypes differentially regulate important physiological processes, such as blood pressure homeostasis, placenta development, mood, and pain perception 6,14 .

The dearth of fully selective ligands for individual aAR subtypes, particularly for $\alpha_{1 \mathrm{~B}} \mathrm{AR}$, aggravates the scarcity of brainpermeable $\alpha A R$ agents to treat neurological and neuropsychiatric disorders ${ }^{3,7-9,15}$. The discovery of subtype-selective compounds is exceptionally challenging due to the high sequence and structural conservation in the orthosteric ligand-binding sites of closely related subtypes, which recognize the same endogenous agonists. In addition, ligand promiscuity often extends to more distant receptor subfamilies because aminergic GPCRs share several key features for ligand recognition ${ }^{16,17}$. For instance, the widely prescribed antipsychotic risperidone and the antidepressant amitriptyline have a high affinity for many aminergic GPCRs, including $\alpha_{1} \mathrm{ARs}^{18}$. Unfortunately, the undesired interaction with $\alpha_{1}$ ARs can result in postural hypotension and related complications as side effects.

Recent high-resolution structures of aminergic GPCRs and a large body of mutagenesis studies have revealed that subtype selectivity can be achieved by exploiting secondary binding pockets, which are less conserved than the orthosteric site ${ }^{16,19-27}$. However, while structures of $\beta A R s$ have been determined bound to various ligands ${ }^{28-34}$, and structures of $\alpha_{2} \mathrm{ARs}$ recently became available $26,35,36$, no $\alpha_{1} \mathrm{AR}$ structure has been reported to date.

In this work, to gain insights into the structure of $a_{1} A R s$ and identify key determinants of ligand selectivity, we determine the crystal structure of human $\alpha_{1 B} A R$ in complex with $(+)$-cyclazosin ${ }^{37-39}$. This inverse agonist shares its piperazinyl quinazoline scaffold with a series of close analogs clinically used as antihypertensive drugs ${ }^{4,5}$ (Supplementary Fig. 1), such as prazosin, doxazosin, and terazosin, which are also currently evaluated in clinical studies to prevent a cytokine storm in COVID-19 patients (https://clinicaltrials.gov/ct2/show/NCT04365257). Remarkably, the piperazinyl quinazoline scaffold is structurally distinct from any other aminergic ligand co-crystallized so far ${ }^{27}$. By comparison with the sequences and structures of $\alpha_{2} \mathrm{ARs}$ and by pharmacological characterization of $\alpha_{1 B} A R-\alpha_{2 C} A R$ chimeras with antagonists and inverse agonists with different selectivity profiles, we identify molecular determinants of selectivity within aARs at positions 3.29 in TM3 and 6.55 in TM6 (Ballesteros-Weinstein numbering ${ }^{40}$ ). Together, our structural and pharmacological analysis provides a basis for the design of novel drugs selectively targeting aAR subtypes, as well as drugs devoid of detrimental off-target interactions with these receptors. Furthermore, the $\alpha_{1 \mathrm{~B}} \mathrm{AR}$ structure presented here may assist the design of fully $\alpha_{1 B} A R$-selective ligands to improve our understanding of this $\alpha_{1} \mathrm{AR}$ subtype's biological roles and explore this receptor as a therapeutic target to treat cardiovascular, neurological, and inflammatory diseases.

\section{Results}

Crystallization and structure determination of $\alpha_{1 B} A R$. As attempts to obtain well-diffracting crystals of a previously stabilized $\alpha_{1 \mathrm{~B}} \mathrm{AR}$ mutant, $\alpha_{1 \mathrm{~B}} \mathrm{AR}-\# 12^{41}$, were unsuccessful, we selected a more stable variant using directed evolution ${ }^{42}$ (Supplementary Table 1). To increase the chances of crystallization, we deleted the $\mathrm{N}$-terminal residues M1-N34 as well as residues K249-L283 in the third intracellular loop (ICL3), and we fused the designed ankyrin repeat protein (DARPin) D12 crystallization chaperone ${ }^{43}$ to the C-terminal end of transmembrane helix 7 (TM7) of the stabilized $\alpha_{1 \mathrm{~B}} \mathrm{AR}$ variant, generating $\alpha_{1 \mathrm{~B}} \mathrm{AR}_{\mathrm{XTAL}}$ (Supplementary Fig. 2). We have recently established the fusion of DARPin D12 to TM7 of a GPCR as a tool to facilitate GPCR crystallization ${ }^{44}$. The stabilizing mutations locked the receptor in a signalinginactive state, as evidenced by the lack of agonist-induced $\mathrm{G}_{\mathrm{q}}$ signaling compared to wild-type $\alpha_{1 B} A R$ (Supplementary Fig. 3a). We observed that the following individual mutations substantially impair agonist-induced $\mathrm{G}_{\mathrm{g}}$ signaling: $\mathrm{S} 95^{2.54} \rightarrow \mathrm{C}$, $\mathrm{S} 150^{34.50} \rightarrow \mathrm{Y}, \quad \mathrm{G} 183^{4.63} \rightarrow \mathrm{V}, \quad \mathrm{D} 191^{\mathrm{ECL}} 2 \rightarrow \mathrm{Y}, \quad \mathrm{T} 295^{6.36} \rightarrow \mathrm{M}$, $\mathrm{V} 333^{7.38} \rightarrow \mathrm{L}, \quad \mathrm{F} 334^{7.39} \rightarrow \mathrm{L}$, and $\mathrm{P} 349^{7.54} \rightarrow \mathrm{L}$ (Supplementary Fig. $3 b, c$ and Supplementary Table 2). We expressed $\alpha_{1 B} A_{X T A L}$ in the inner membrane of $E$. coli and isolated properly folded receptors via a prazosin ligand-affinity column (see "Methods").

The inverse agonist cyclazosin is a racemic mixture of the $(+)$ and (-)-enantiomers (Fig. 1a). We use the term cyclazosin to refer to this racemic mixture, and we specify which particular enantiomer where required. The affinity of cyclazosin for $\alpha_{1 B} A_{X T A L}$ is high and only marginally reduced compared to wild-type $\alpha_{1 \mathrm{~B}} \mathrm{AR} \quad\left(K_{\mathrm{i}}=6.17 \mathrm{nM}\right.$ and $1.02 \mathrm{nM}$, respectively) (Supplementary Fig. 3d, e). The fusion of DARPin D12 did not significantly perturb cyclazosin affinity. Cyclazosin-bound $a_{1 B} A_{X T A L}$ exhibited high thermostability in a CPM assay ${ }^{45}$ (apparent $T_{\mathrm{m}} \approx 71^{\circ} \mathrm{C}$ ) (Supplementary Fig. 3f). This complex was thus suitable for crystallization in the lipidic cubic phase (LCP).

We co-crystallized $\alpha_{1 \mathrm{~B}} \mathrm{AR}_{\mathrm{XTAL}}$ with cyclazosin in LCP and determined its structure (Fig. 1b, Supplementary Figs. 2b and 4, and Supplementary Table 3). Crystallization of $a_{1 B} A R_{X T A L}$ in the presence of cyclazosin yielded only a limited number of useful crystals. One hundred and nine partial datasets were recorded, carefully inspected, and bad data regions removed (see "Methods"). Data reduction of the best 27 morphologically close data wedges (between $15^{\circ}$ and $32^{\circ}$ ), selected for the similarity in their unit cell parameters, resulted in a $98 \%$ complete dataset at $3.1 \AA$ resolution. To correct for the moderate anisotropy of the data 
a

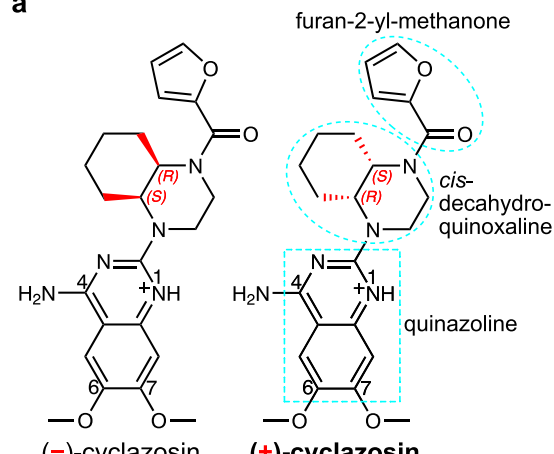

$(-)$-cyclazosin

$(+)$-cyclazosin

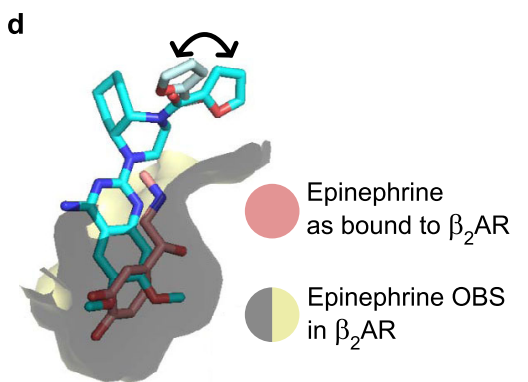

b

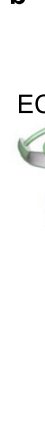

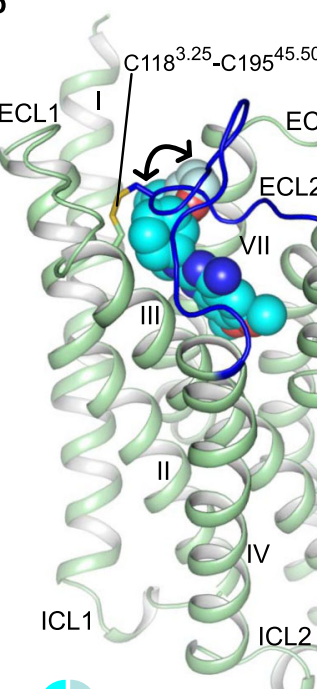

$(+)$-cyclazosin

$\alpha_{1 \mathrm{~B}} \mathrm{AR} \mathrm{R}_{\mathrm{XTAL}}$
13

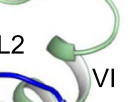

(1)

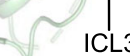

CL3

c

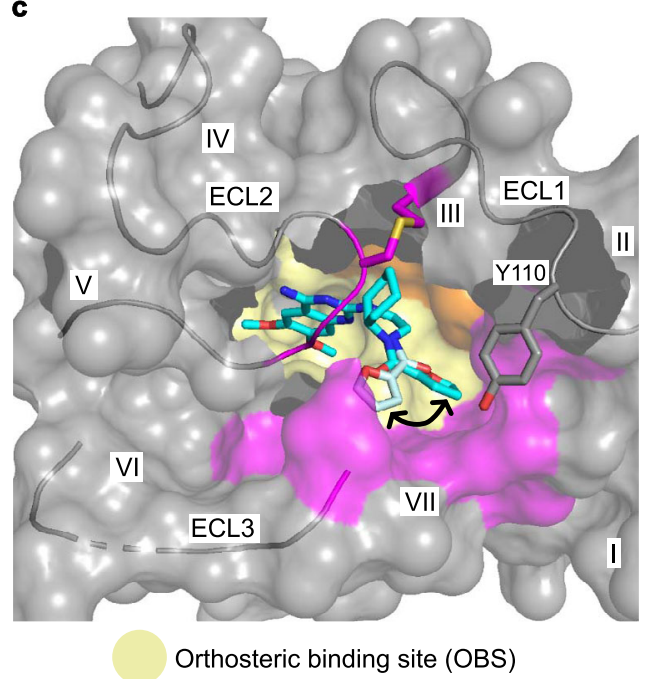

Secondary binding pockets (SBPs)

Boundary OBS/SBPS

$\curvearrowleft$ Two orientations of furan-2-yl-methanone

Fig. 1 Structure of $\alpha_{1 \mathbf{B}} \mathbf{A} \mathbf{R}_{\mathbf{X T A L}}$ bound to ( + )-cyclazosin and overview of the ligand-binding site. a Chemical structure of $(-)$ - and $(+)$-cyclazosin. $\mathrm{N} 1$ is expected to be mostly protonated at the crystallization $\mathrm{pH}$ of 6.0 (see "Methods") as well as at physiological pH, and the resulting positive charge will be delocalized over the quinazoline ring system ${ }^{49-51}$. b Structure of $\alpha_{1 B} A R_{X T A L}$ bound to (+)-cyclazosin. For clarity, DARPin D12 has been omitted. $(+)$-Cyclazosin is depicted as van der Waals spheres. The two orientations observed for the furan-2-yl-methanone substituent of (+)-cyclazosin are colored in cyan and pale cyan, respectively, and are indicated by a black curved arrow. Oxygen, nitrogen, and sulfur atoms are depicted in red, blue, and yellow, respectively. ECL, extracellular loop; ICL, intracellular loop. c Surface representation of the (+)-cyclazosin binding site in $\alpha_{1 B} A R_{X T A L}$. ECL1-3 are shown as surface and as cartoon; (+)-cyclazosin is shown as sticks. The orthosteric binding site (OBS) has been approximated on the basis of the $\beta_{2} A R$-epinephrine complex (see panel $d$ and main text). $\mathbf{d}$ Comparison of the binding modes of $(+)$-cyclazosin in $\alpha_{1 B} A R_{X T A L}$ and epinephrine in $\beta_{2} A R$ (PDB ID: $4 \mathrm{LDO}^{30}$ ).

(deltaB $\approx 17 \AA^{2}$ ), we submitted our unmerged data to the STARANISO server ${ }^{46}$. The result was an anisotropy-corrected dataset extending to $2.87 \AA$ despite low completeness and poor data collection statistics in the highest resolution shell (Supplementary Table 3). Refinement of the cyclazosin- $\alpha_{1 B} A_{\mathrm{XTAL}}$ complex was performed with both the $3.1-\AA$ and $2.87-\AA$ datasets and yielded very similar structures. However, better electron density maps and refinement statistics were obtained with the 2.87- $\AA$ anisotropy-corrected dataset. The electron density was of good quality, except for the following receptor regions: residues $35-37$ of the truncated $\mathrm{N}$ terminus, $238^{5.73}-247^{\mathrm{ICL} 3}$ of the shortened ICL3, and 3206.61-323 ECL3 (Ballesteros-Weinstein numbering 40 denoted in superscript).

Strong electron density in the ligand-binding pocket allowed unambiguous modeling of $(+)$-cyclazosin and key receptor side chains discussed herein (Supplementary Fig. 5), except V197 45.52 in ECL2. Despite the use of racemic cyclazosin and the fact that both enantiomers have a similar high affinity for wild-type $a_{1 B} A^{37}$, binding of the $(+)$-enantiomer was favored in our crystals. Two distinct orientations of the furan-2-yl-methanone substituent of $(+)$-cyclazosin were observed (Fig. 1b, c and Supplementary Fig. 5a, b), which indicates a certain degree of conformational freedom for this moiety in $\alpha_{1 \mathrm{~B}} \mathrm{AR}_{\mathrm{XTAL}}$.

Architecture of $\boldsymbol{\alpha}_{1 \mathrm{~B}} \mathrm{AR}_{\mathrm{XTAL}} \cdot \alpha_{1 \mathrm{~B}} \mathrm{AR}_{\mathrm{XTAL}}$ in complex with $(+)$-cyclazosin exhibits the canonical GPCR architecture consisting of TM1-7 connected by three intracellular loops (ICL1-3) and three extracellular loops (ECL1-3) (Fig. 1b). Helix 8 has been replaced by the DARPin D12 fusion (Supplementary Fig. 2). The ECLs lack regular secondary structure (Fig. 1b). ECL2 is tethered to the extracellular tip of TM3 through the conserved disulfide bridge between $\mathrm{C} 195^{45.50}$ and $\mathrm{C} 118^{3.25}$, forming a partial lid on the $(+)$-cyclazosin binding site (Fig. 1b, c). We note, however, that crystal contacts are formed by ECL2 (Supplementary Fig. 4d).

Except for TM1, which is tilted outwards as a consequence of crystal packing (Supplementary Fig. 4d, e), the seventransmembrane (7TM) bundle of $a_{1 \mathrm{~B}} \mathrm{AR}_{\mathrm{XTAL}}$ adopts a similar arrangement as observed in other antagonist-bound ARs, including $\alpha_{2 \mathrm{~A}} \mathrm{AR}$ and $\alpha_{2 \mathrm{C}} \mathrm{AR}$ bound to the antagonist RS79948 (PDB IDs: $6 \mathrm{KUX}^{35}$ and $6 \mathrm{KUW}^{26}$, respectively) and $\beta_{1} \mathrm{AR}$ and $\beta_{2}$ AR bound to the antagonist carazolol (PDB IDs: 2 YCW $^{32}$ and $2 \mathrm{RH}^{28}$, respectively) (Supplementary Figs. 6 and 7). $\alpha_{1 \mathrm{~B}} \mathrm{AR}_{\mathrm{XTAL}}$ apparently captured an inactive state, as evidenced by the closed intracellular arrangement of TM6 (Supplementary Fig. 6) and the negative activation index $\left(\mathrm{A}^{100}=-45.7\right)^{47}$. The nearly identical arrangement of the cytoplasmic end of TM7 in $\alpha_{1 B} A_{X T A L}$ compared to the other inactive-state AR structures suggests that the DARPin D12 fusion did not perturb its conformation. The extracellular end of TM3 in $\alpha_{1 B} A_{\mathrm{XTAL}}$ is more inward-pointing compared to the above-mentioned $\alpha_{2} A R$ and $\beta A R$ structures (Supplementary Fig. 7). TM4 is over one helical turn longer in $a_{1 \mathrm{~B}} \mathrm{AR}_{\mathrm{XTAL}}$ compared to the $\alpha_{2} \mathrm{AR}$ structures, and thus, it resembles the $\beta A R$ structures (Supplementary Fig. 7). The shorter TM4 observed in the $\alpha_{2} \mathrm{AR}$ structures is likely the consequence of two consecutive proline residues at its extracellular end. The lack of regular secondary structure in ECL2 of $\alpha_{1 \mathrm{~B}} \mathrm{AR}_{\mathrm{XTAL}}$ resembles the $\alpha_{2} \mathrm{AR}$ structures, whereas in $\beta$ ARs ECL2 forms a short $\alpha$-helix. Of note, K331 3.36 establishes a salt bridge with E106 2.65 in $a_{1 B} A_{\text {XTAL }}$ (Supplementary Fig. 7a). Mutations abolishing the positive charge of $\mathrm{K} 331^{7.36}$ resulted in constitutive receptor 
activity $^{48}$; however, whether the K331 3.36 -E106 ${ }^{2.65}$ salt bridge is involved in receptor activation requires further studies.

$(+)$-Cyclazosin extends from the orthosteric binding site toward secondary binding pockets. (+)-Cyclazosin has a reported 100-1,000-fold selectivity for $\alpha_{1}$ ARs over $\alpha_{2}$ ARs, and a slight preference for $\alpha_{1 B} A R$ over the other $\alpha_{1} A R$ subtypes ${ }^{37}$. To identify the determinants of ligand selectivity and to guide compound optimization, we first inspected the binding mode of $(+)$-cyclazosin in $\alpha_{1 B} A R_{X T A L}$, followed by sequence and structural comparisons with the closely related $\alpha_{2}$ ARs.

$(+)$-Cyclazosin adopts an inverted L-shaped binding mode in $a_{1 B} A_{X T A L}$ (Fig. 1c). The dimethoxyquinazoline moiety inserts deeply into the binding pocket, occupying the orthosteric binding site (OBS), i.e., the site that accommodates the endogenous agonists epinephrine and norepinephrine. As there is currently no structure of an aAR in complex with an endogenous agonist, the OBS was approximated on the basis of the $\beta_{2} \mathrm{AR}$-epinephrine complex (Fig. 1d) (PDB ID: $4 \mathrm{LDO}^{30}$ ), in agreement with mutagenesis studies defining a common OBS within $\mathrm{ARs}^{4}$. The cis-decahydroquinoxaline moiety of $(+)$-cyclazosin, composed of a piperazine and a fused cyclohexane ring (Fig. 1a), is accommodated at the boundary between the OBS and a secondary binding pocket defined by TM3 and ECL2 (Fig. 1c). Finally, the two distinct conformations adopted by the furan-2-yl-methanone moiety are accommodated in secondary binding pockets proximal to the extracellular surface (Fig. 1c). (+)-Cyclazosin can be thus considered a "bitopic" ligand that fills simultaneously both the OBS and secondary binding pockets.

Twenty-five residues delineate the ligand-binding pocket of

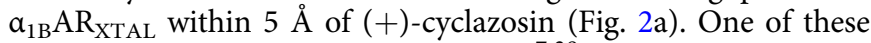
residues is the stabilizing mutation $\mathrm{F} 334^{7.39} \rightarrow \mathrm{L}$, which is adjacent to the $\mathrm{V} 333^{7.38} \rightarrow \mathrm{L}$ mutation. Upon back-mutation of $\mathrm{L} 334^{7.39}$ to the wild-type phenylalanine, we observed a loss of thermostability (Supplementary Fig. 3f and ref. ${ }^{41}$ ), and no crystals could be obtained despite extensive efforts. The back-mutations $\mathrm{L} 334^{7.39} \rightarrow \mathrm{F}$ and $\mathrm{L} 333^{7.38} \rightarrow \mathrm{V}$ were thus modeled into the structure of $\alpha_{1 B} A R_{X T A L}$, and molecular dynamics (MD) simulations were carried out on both this model as well as on the crystal structure (i.e., without back-mutations) in a lipid bilayer. Throughout these simulations, the receptor and the ligand exhibited structural stability, as evidenced by the root-meansquare deviation (RMSD) of the protein backbone (especially TM2-7, containing nearly all ligand-interacting residues) and ligand heavy atoms, respectively (Supplementary Fig. 8a, b). The simulations confirmed the ligand-binding mode observed in the crystal structure (Fig. 2 b and Supplementary Fig. 8). F334 ${ }_{\mathrm{MD}}{ }^{7.39}$ favored a gauche minus $\left(g^{-}\right) \chi_{1}$ rotameric state (Fig. $2 b$ ) and resulted in stable binding of $(+)$-cyclazosin through aromatic contacts with the quinazoline and furan rings (Supplementary Fig. 8c, d). The L334 $4^{7.39} \rightarrow$ F back-mutation did not perturb the conformation of the $\mathrm{Y} 338^{7.43}$ side chain or the hydrogen bond between the latter side chain and D125.32 observed in $a_{1 \mathrm{~B}} \mathrm{AR}_{\mathrm{XTAL}}$ (Fig. 2a, b and Supplementary Fig. 8e), which stabilizes the binding pocket ${ }^{16}$, nor did it affect the furan moiety of $(+)$-cyclazosin (Supplementary Fig. 8f, g).

The side chain of D1253.32 in $a_{1 \mathrm{~B}} \mathrm{AR}$ XTAL forms a chargereinforced hydrogen bond with $\mathrm{N} 1$ of $(+)$-cyclazosin (Fig. 2a, c), which is expected to be protonated and the resulting positive charge delocalized over the quinazoline ring system ${ }^{49-51}$ (ligandreceptor interactions are summarized in Supplementary Table 4). On one side of the pocket, the dimethoxyquinazoline moiety faces a hydrophobic patch in TM6 and TM7, consisting of W3076.48, $\mathrm{F} 310^{6.51}, \mathrm{~F} 311^{6.52}, \mathrm{~L} 314^{6.55}$, and $\mathrm{F} 334_{\mathrm{MD}}{ }^{7.39}$ (Fig. $2 \mathrm{a}-\mathrm{c}$ ). On the opposite side of the pocket, the quinazoline ring establishes van der Waals contacts with A122 $2^{3.29}$ and V126 3.33 . The cyclohexane ring of the cis-decahydroquinoxaline moiety protrudes toward TM3 and ECL2, and establishes van der Waals contacts with $\mathrm{W} 121^{3.28}$ and $\mathrm{A} 122^{3.29}$. In one of the two modeled orientations, the furan ring inserts in a hydrophobic secondary pocket between TM2, TM3, and TM7, establishing van der Waals contacts with $\mathrm{L} 105^{2.64}$, W $121^{3.28}$, W335 ${ }^{7.40}$, and $\mathrm{F} 334_{\mathrm{MD}}{ }^{7.39}$, complemented by aromatic interactions with the latter three residues (Fig. $2 \mathrm{a}-\mathrm{c}$ ). In the alternative orientation, the furan ring points toward D $327^{7.32}$, F330 7.35 , and $\mathrm{V} 197^{45.52}$ (Fig. 2a).

Overall, the dimethoxyquinazoline moiety of $(+)$-cyclazosin is tightly anchored within the conserved epinephrine/norepinephrine OBS, while the remaining parts of this ligand are accommodated in distinct secondary binding pockets (SBPs) or at the boundary between OBS and SBPs (Fig. 1c). The subpockets shaping the OBS and SBPs may offer opportunities for ligand optimization; however, a detailed understanding of their role in ligand recognition among closely related receptors is required, which we discuss next.

Comparison of ligand-binding pockets between $\alpha_{1} \mathrm{ARs}$ and $\boldsymbol{a}_{2}$ ARs. As mentioned above, $(+)$-cyclazosin preferentially binds to $a_{1}$ ARs over $\alpha_{2} \mathrm{ARs}^{37}$. In contrast, the antagonist RS79948 exhibits a remarkable $\sim 10,000$-fold selectivity for $\alpha_{2}$ ARs over $\alpha_{1}$ ARs ${ }^{18,52,53}$. To identify key determinants of ligand selectivity in aARs, we compared the binding sites of $(+)$-cyclazosin in $\alpha_{1 B} A_{X T A L}$ and RS79948 in $\alpha_{2 \mathrm{C}} \mathrm{AR}$ (PDB ID: 6KUW ${ }^{26}$ ).

This comparison revealed seven non-conserved residues between $\alpha_{1 \mathrm{~B}} \mathrm{AR}$ and $\alpha_{2 \mathrm{C}} \mathrm{AR}$ (Fig. 3a), which correspond to positions $2.64,3.28,3.29,45.52,5.43,6.55$, and 7.32. Positions 45.52 and 5.43 are only partially non-conserved among all six aARs (Fig. 3a). We then superposed the structure of $\alpha_{1 B} A R_{X T A L}$ bound to $(+)$-cyclazosin with the $\alpha_{2 C}$ AR-RS79948 complex and inspected the above-mentioned non-conserved residues (Fig. $3 \mathrm{~b}$ ) (superposition of the conserved residues within the ligandbinding sites is shown in Supplementary Fig. 9). Four of the nonconserved residues form direct interactions with $(+)$-cyclazosin in $\alpha_{1 B} A R_{X T A L}$, and these residues are $\mathrm{L} 105^{2.64}, \mathrm{~W} 121^{3.28}$, A122 $2^{3.29}$, and L314 ${ }^{6.55}$. Similarly, four of the non-conserved residues form direct interactions with RS79948 in $\alpha_{2 C} \mathrm{AR}$, and these residues are Y1273.28, L128 $8^{3.29}, \mathrm{~L} 204^{45.52}$, and Y402 ${ }^{6.55}$. To assess the impact of these residues on selective ligand recognition, we made chimeric $a_{1 B} A R-a_{2 C} A R$ mutants. For this purpose, we converted the residues in $\alpha_{1 B} A R$ at the above-mentioned positions to the corresponding $\alpha_{2 C} A R$ residues, either individually or in combination, and we assessed the effect on ligand affinities. We refer to the chimeric $\alpha_{1 B} A R-\alpha_{2 C} A R$ mutants with the term " $\alpha_{1 B} A R-a_{2 C}$ " and specify in parentheses the chimeric modification. For example, $\alpha_{1 B} \mathrm{AR}-\alpha_{2 \mathrm{C}}\left(\mathrm{L}^{3.29}\right)$ corresponds to $a_{1 \mathrm{~B}} \mathrm{AR}$ bearing the $\mathrm{A} 122^{3.29} \rightarrow \mathrm{L}$ chimeric substitution.

Molecular determinants of selective ligand binding to $\alpha_{2} \mathrm{ARs}$ over $\boldsymbol{\alpha}_{\mathbf{1}}$ ARs. We started our analysis with RS79948, as it exhibits a higher selectivity ratio $\left[\alpha_{2}: \alpha_{1}\right.$ ARs $\approx 10,000$ (refs. ${ }^{18,53}$ )] than $(+)$-cyclazosin $\left[\alpha_{1}: \alpha_{2}\right.$ ARs $\approx 100-1000$ (ref. $\left.\left.{ }^{37}\right)\right]$. We compared the affinity of RS79948 for the chimeric $\alpha_{1 B} A R-\alpha_{2 C}$ mutants to that of wild-type $\alpha_{1 B} A R$ and $\alpha_{2 C} A R$ (Fig. $4 a$, Supplementary Fig. 10, and Supplementary Table 6). In agreement with previous studies $^{18,53}$, RS79948 had sub-nM affinity for $\alpha_{2 C} A R$ and only $\mu M$ affinity for $\alpha_{1 \mathrm{~B}} \mathrm{AR}$. Strikingly, residue 3.29 was found to be key for RS79948 binding, as the A122 $2^{3.29} \rightarrow \mathrm{L}$ chimeric substitution in $\alpha_{1 \mathrm{~B}} \mathrm{AR}$ improved the affinity of this ligand by a remarkable 140 fold. $\mathrm{L} 314^{6.55} \rightarrow \mathrm{Y}$ and $\mathrm{V} 197^{45.52} \rightarrow \mathrm{L}$ resulted in 6 - and 2 -fold higher affinity, respectively, whereas the apparent slight improvement observed for $\mathrm{W} 121^{3.28} \rightarrow \mathrm{Y}$ did not reach statistical 


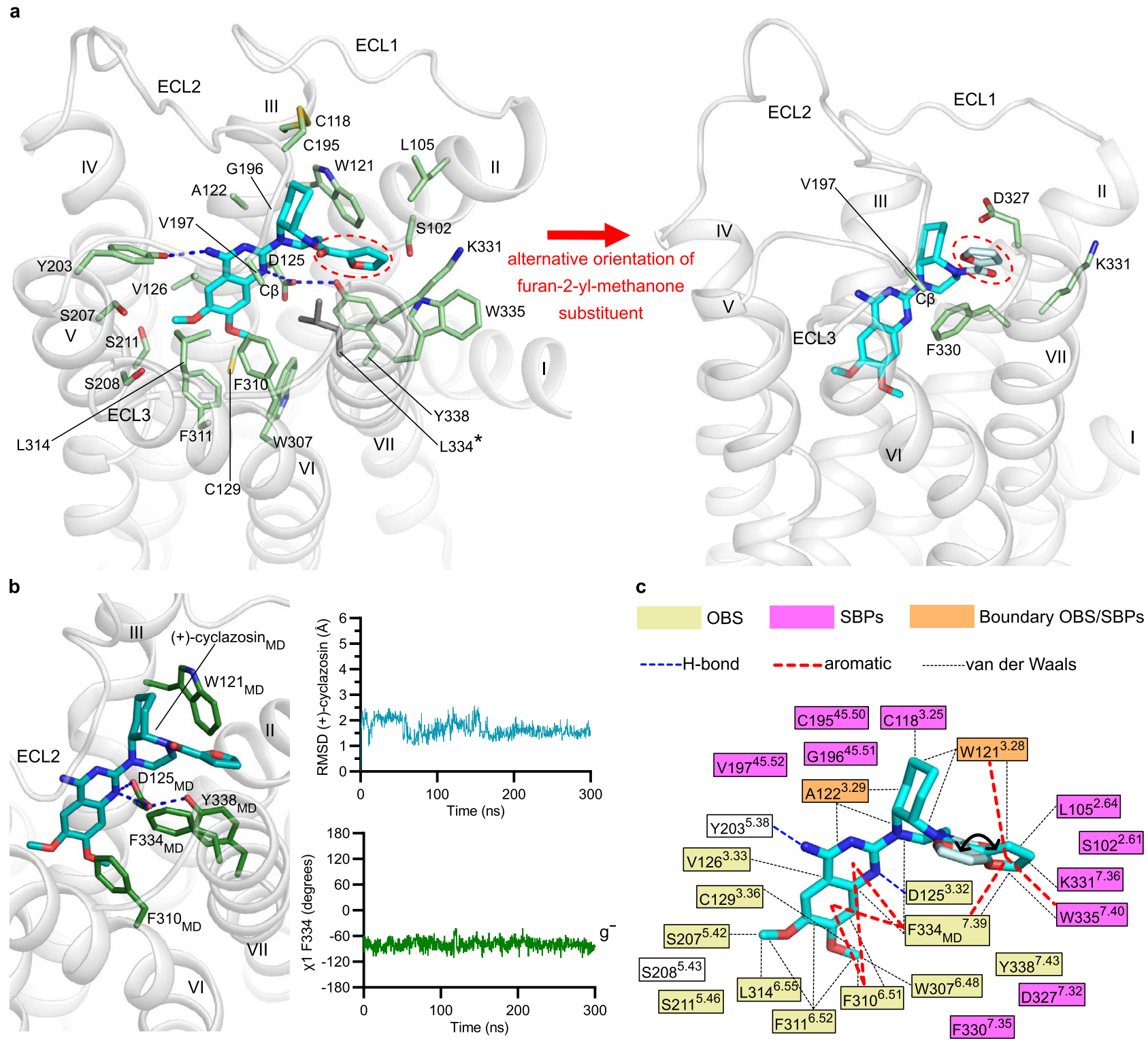

Fig. $2(+)$-Cyclazosin binding pocket in $\boldsymbol{\alpha}_{\mathbf{1 B}} \mathbf{A} \mathbf{R}_{\mathbf{X T A L}}$ - a Detailed view of the $(+)$-cyclazosin binding site. ( + )-Cyclazosin is shown as sticks in cyan, with the two alternative orientations observed for the furan-2-yl-methanone substituent (highlighted by a dashed red ellipse) colored in cyan (on the left) and pale cyan (on the right), respectively. Receptor residues are shown as sticks in pale green except for the F334 $\rightarrow \mathrm{L}$ mutation, which is colored in dark gray and is indicated by an asterisk. V197 is shown to $C \beta$ only because its side chain is not resolved in the electron density map. Hydrogen bonds are depicted as dashed blue lines. Oxygen, nitrogen, and sulfur atoms are depicted in red, blue, and yellow, respectively. b MD simulation of $\alpha_{1 B} A R_{X T A L-M D}-V 333-F 334$. The plots on the right indicate the structural stability of $(+)$-cyclazosin and F334 $\mathrm{MD}$ throughout the simulation. RMSD, root-mean-square deviation; $\mathrm{g}^{-}$, gauche minus conformation of the $\chi_{1}$ dihedral angle. For $(+)$-cyclazosin, RMSD values were calculated on all atoms. A representative snapshot of the final nanosecond of the simulation is depicted on the left, viewed from the same perspective as in panel a. (+)-Cyclazosin is colored in teal; F334 $\mathrm{MD}_{\mathrm{D}}$ is colored in dark green. c Schematic representation of the (+)-cyclazosin binding site. OBS, orthosteric binding site; SBPs, secondary binding pockets. A black curved arrow indicates the two orientations observed for the furan-2-yl-methanone moiety. Note that residues C19545.50, G19645.51, and V19745.52 belong to ECL2, which forms crystal contacts. Source data are provided as a Source Data file.

significance (Supplementary Table 7). The remarkable impact of $\mathrm{A} 122^{3.29} \rightarrow \mathrm{L}$ in $\alpha_{1 \mathrm{~B}} \mathrm{AR}$ is consistent with the observation that the reciprocal $\mathrm{L} 128^{3.29} \rightarrow \mathrm{A}$ chimeric substitution in $\alpha_{2 \mathrm{C}} \mathrm{AR}$ decreases the ability of RS79948 to antagonize agonist-induced signaling by 50 -fold ${ }^{26}$. We also made an $\alpha_{1 B} A R$ mutant bearing all four chimeric substitutions, i.e., $\mathrm{W} 121^{3.28} \rightarrow \mathrm{Y}, \mathrm{A} 122^{3.29} \rightarrow \mathrm{L}$, $\mathrm{V} 197^{45.52} \rightarrow \mathrm{L}$, and $\mathrm{L} 314^{6.55} \rightarrow \mathrm{Y}-$ termed $\alpha_{1 \mathrm{~B}} \mathrm{AR}-\alpha_{2 \mathrm{C}}$ (YLLY). The affinity of RS79948 for $\alpha_{1 B}$ AR- $\alpha_{2 C}$ (YLLY) was improved by almost three orders of magnitude compared to wild-type $\alpha_{1 \mathrm{~B}} \mathrm{AR}$ (Fig. 4a), approaching the affinity for $\alpha_{2 C} A R$. Compared to the $\mathrm{A} 122^{3.29} \rightarrow \mathrm{L}$ chimeric substitution alone, the additional gain of affinity observed for $\alpha_{1 B} A R-\alpha_{2 C}$ (YLLY) corresponded to an $\sim 5$-fold improvement. No further improvement in the affinity of RS79948 was observed by introducing chimeric substitutions at the remaining three non-conserved positions within the binding site of $\alpha_{1 B} A R$, i.e., $L 105^{2.64} \rightarrow \mathrm{N}, \mathrm{S} 208^{5.43} \rightarrow \mathrm{C}$, and $\mathrm{D} 327^{7.32} \rightarrow \mathrm{G}$ (Supplementary Table 6). We also replaced the entire ECL2 in $a_{1 B} A R-a_{2 C}$ (YLLY) with the corresponding $a_{2 C} A R$ sequence (from $\mathrm{I}_{178^{4.58}}$ to $\mathrm{F} 202^{5.37}$, i.e., including the tips of TM4 and TM5), generating $\alpha_{1 \mathrm{~B}} \mathrm{AR}-\alpha_{2 \mathrm{C}}$ (YLLY;ECL2). However, no further improvement in RS79948 affinity was observed upon the replacement of ECL2 (Supplementary Table 6). 


\begin{tabular}{|c|c|c|c|c|c|c|c|c|c|c|c|c|c|c|c|c|c|c|c|c|c|c|c|}
\hline & \multicolumn{23}{|c|}{ Ballesteros-Weinstein } \\
\hline & 2.61 & 2.64 & 3.25 & 3.28 & 3.29 & 3.32 & 3.33 & 3.36 & 3.37 & $45.50,-\overline{45.51}$ & $4 \overline{45.52} ; 5.38$ & $5.42 !$ & 5.43 & 5.46 & 6.48 & 6.51 & 6.52 & 6.55 & 7.32 & $7.35,7.36$ & 7.39 & 7.40 & 7.43 \\
\hline$\alpha_{1 \mathrm{~A}}$ & $\mathbf{s}$ & $F$ & c & w & A & D & v & C & $T$ & ia & 1 & $\mathbf{s}$ & :A & is & w & $\mathbf{F}$ & $\mathbf{F}$ & M & E & $\mathbf{F}$ & & & $\mathbf{Y}$ \\
\hline$\alpha_{1 B}$ & S102 & L105 & C118 & W121 & A122 & D125 & V126 & C 129 & $\mathrm{~T} 130$ & C195'G196 & V197: Y 203 & S207i & zis208! & is 211 & W $\underline{307}$ & $F 310$ & $F$ & $L 314$ & |D327 | & F330i K331i & $F \underline{334}$ & W 335 & 5 Y Y338 \\
\hline$\alpha_{1 D}$ & s & M & c & w & A & D & v & c & T & c IG & $\begin{array}{l:l} & y\end{array}$ & s 1 & is & is & w & $\mathbf{F}$ & $\mathbf{F}$ & L & E & F ik & if & w & $\mathbf{Y}$ \\
\hline$\alpha_{2 A}$ & $\mathrm{~s}$ & N & c & Y & L & D & v & c & $T$ & c & 'Y & $\mathrm{s}$ & ic & is & w & $\mathrm{F}$ & $\mathbf{F}$ & $\mathrm{Y}$ & R & ; $k$ & $F$ & w & Y \\
\hline$\alpha_{2 B}$ & s & N & c & $Y$ & L & D & v & c & $T$ & $\mathrm{k}$ & iy & s & is & is & w & $\mathrm{F}$ & $\mathbf{F}$ & $\mathrm{Y}$ & H & $Q$ & $\mathrm{~F}$ & w & Y \\
\hline$\alpha_{2 C}$ & S108 & N111 & C124 & $\mathrm{Y} 127$ & $L 128$ & D131 & V132 & $\mathrm{C} 135$ & T136 & C202/G203 & $\begin{array}{ll:l}L 204 & Y 210\end{array}$ & S214i & $+C 215$ & $\$ 218$ & W 395 & $F 398$ & F 399 & $\mathrm{Y} 402$ & G416 & $\mathbf{F} 419$ & $F \underline{423}$ & W424 & $4 \mathbf{Y} 427$ \\
\hline
\end{tabular}

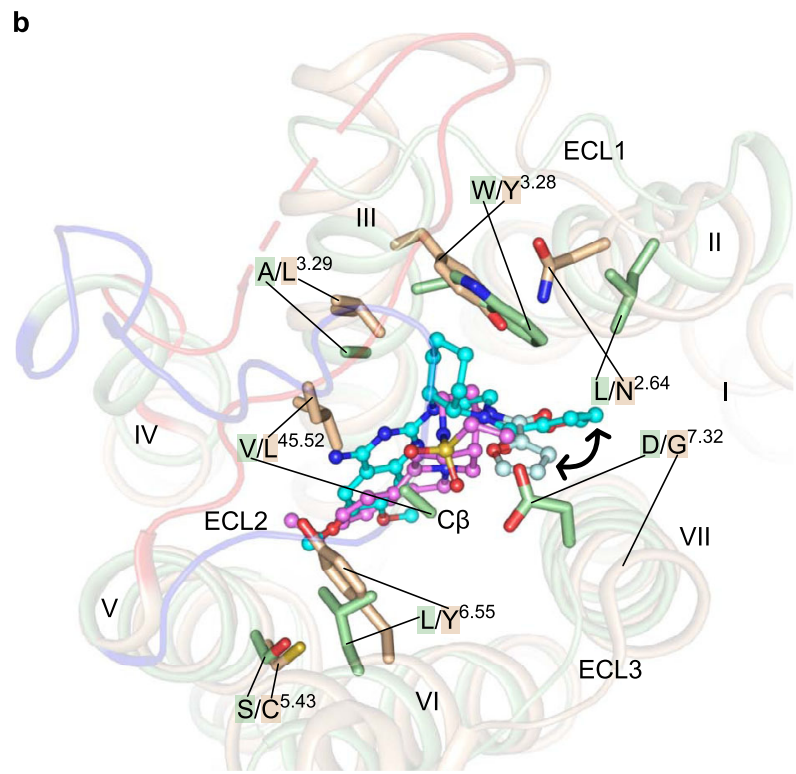

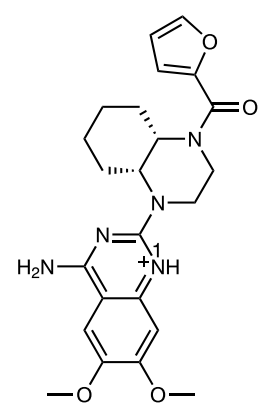

$(+)$-cyclazosin

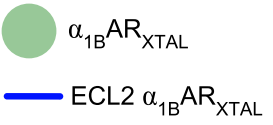

$(+)$-cyclazosin

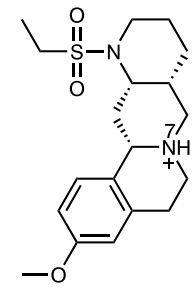

RS79948

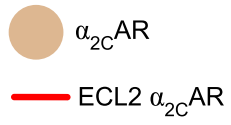

RS79948

Fig. 3 Comparison of the ligand-binding pockets of $\alpha_{1 B} \mathbf{A R}$ XTAL bound to ( + -)-cyclazosin and $\alpha_{2} \mathbf{A R}-\mathbf{R S 7 9 9 4 8}$. a Alignment of residues delineating the binding pockets of $(+)$-cyclazosin in $\alpha_{1 B}$ ARXTAL and of RS79948 in $\alpha_{2}$ AR (PDB ID: 6KUW ${ }^{26}$ ). Non-conserved residues between human $\alpha_{1} A R s$ and $\alpha_{2}$ ARs are highlighted by solid blue rectangles, whereas dashed blue rectangles highlight partially non-conserved residues. Underlined black residues interact with the cognate ligand, whereas non-underlined black residues do not, but are nonetheless within $5 \AA$ of it (ligand-receptor interactions are listed in Supplementary Tables 4 and 5). Gray residues are $>5 \AA$ away from the cognate ligand. Aromatic residues are highlighted in orange, hydrophobic residues in yellow, polar residues in green, Cys in yellow-green, acidic residues in red, basic residues in blue. $\mathbf{b}$ Superposition of $\alpha_{1 B} A R_{X T A L}$ bound to (+)-cyclazosin with $\alpha_{2 C}$ AR-RS79948, focusing on the non-conserved residues within the ligand-binding pocket (cf. panel a). Receptor residues are shown as sticks; ligands are shown in ball-and-stick representation. V197 is shown to $C \beta$ only because its side chain is not resolved in the electron density map. A black curved arrow indicates the two orientations observed for the furan-2-yl-methanone substituent of (+)-cyclazosin. Oxygen, nitrogen, and sulfur atoms are depicted in red, blue, and yellow, respectively.

Overall, our data indicate that the presence of L128 3.29 and $\mathrm{Y} 402^{6.55}$ in $\alpha_{2 \mathrm{C}} \mathrm{AR}$, compared to A122 3.29 and $\mathrm{L} 314^{6.55}$ in $\alpha_{1 \mathrm{~B}} \mathrm{AR}$, is the main reason for the high $\alpha_{2 C}$ AR selectivity of RS79948. The importance of residues 3.29 and 6.55 for the selectivity profile of RS79948 can most likely be extended to all aARs, owing to the conservation of $\mathrm{A}^{3.29}$ within $\alpha_{1}$ ARs and of $\mathrm{L}^{3.29}$ and $\mathrm{Y}^{6.55}$ within $\alpha_{2}$ ARs (Fig. 3a). Position 6.55 deviates within $\alpha_{1}$ ARs in $\alpha_{1 A} A R$. In this subtype, residue 6.55 is methionine, which is, however, a large aliphatic residue as $L^{6.55}$ in $\alpha_{1 B} A R$ and $\alpha_{1 D} A R$ (Fig. 3a).

Structural basis for $\boldsymbol{\alpha}_{2} \mathrm{AR}$-selective ligand recognition. Inspection of the above-mentioned non-conserved residues in the structures of $\alpha_{1 \mathrm{~B}} \mathrm{AR}_{\mathrm{XTAL}}$ bound to (+)-cyclazosin and $\alpha_{2 \mathrm{C}} \mathrm{AR}$ bound to RS79948 (PDB ID: $6 \mathrm{KUW}^{26}$ ) provides a rationale for their role in ligand selectivity (Figs. $3 b$ and $4 b, c)$. In $\alpha_{2 C} A R$, L128 ${ }^{3.29}$ stabilizes the position of RS79948 close to TM3 through hydrophobic contacts with the polycyclic ring system of this antagonist. This positional stabilization may favor the crucial interaction between the side chain of D1313.32 in TM3 and the ligand's N7 atom, which is expected to be protonated (Fig. 4b). In addition, L128 ${ }^{3.29}$ stabilizes the position of L204 ${ }^{45.52}$ in ECL2 through hydrophobic contacts. In turn,
L2045.52 restricts the binding site and establishes hydrophobic interactions with RS79948.

The corresponding residue in $\alpha_{1 \mathrm{~B}} \mathrm{AR}, \mathrm{A} 122^{3.29}$, may not form sufficiently strong contacts, neither with RS79948 nor with residues in ECL2 (Figs. 3b and 4c), resulting in weaker binding of this ligand. The conformation adopted by ECL2 also substantially differs between the two complexes (Figs. $3 \mathrm{~b}$ and $4 \mathrm{c}$ ), although this should be interpreted with caution because these structures are bound to different ligands, and crystal contacts are established by ECL2. Nonetheless, the different conformation adopted by ECL2 on top of the binding sites in $\alpha_{1 B} A R$ and $\alpha_{2 C} A R$ suggests a role for this region in ligand selectivity. As the course of ECL2 is influenced by contacts with other receptor regions, it is difficult to reproduce its effect on ligand selectivity using chimeras.

We also note an inward tilting of the extracellular end of TM3 in $\alpha_{1 B} A_{\mathrm{XTAL}}$ compared to the $\alpha_{2 \mathrm{C}} \mathrm{AR}-\mathrm{RS} 79948$ complex, as well as a different rotamer adopted by the side chain of $\mathrm{D}^{3.32}$ (Fig. 4c). The ligand-binding pocket is thus narrower in $\alpha_{1 \mathrm{~B}} \mathrm{AR}_{\mathrm{XTAL}}$ in this region, which may preclude RS79948 from binding deeply in the TM bundle. Thus, depending on the plasticity of this receptor region, the shape of the pocket in the proximity of $\mathrm{D}^{3.32}$ will possibly contribute to ligand selectivity. 
a

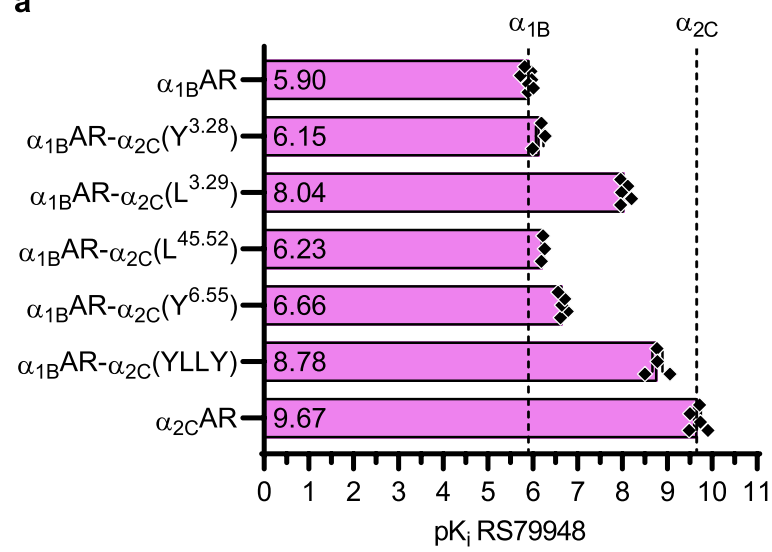

c

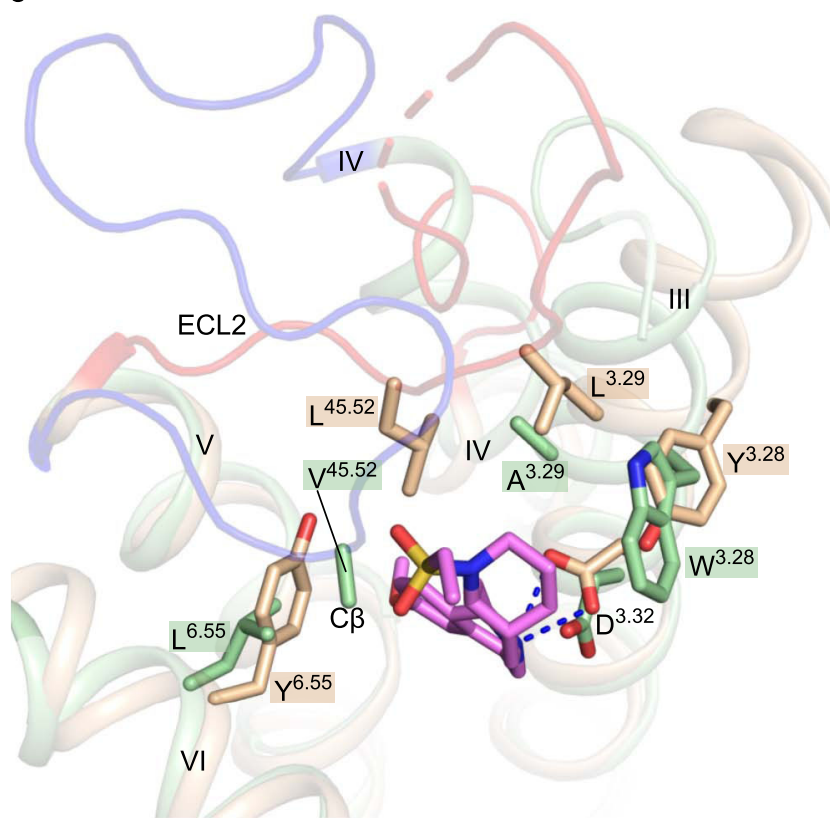

b

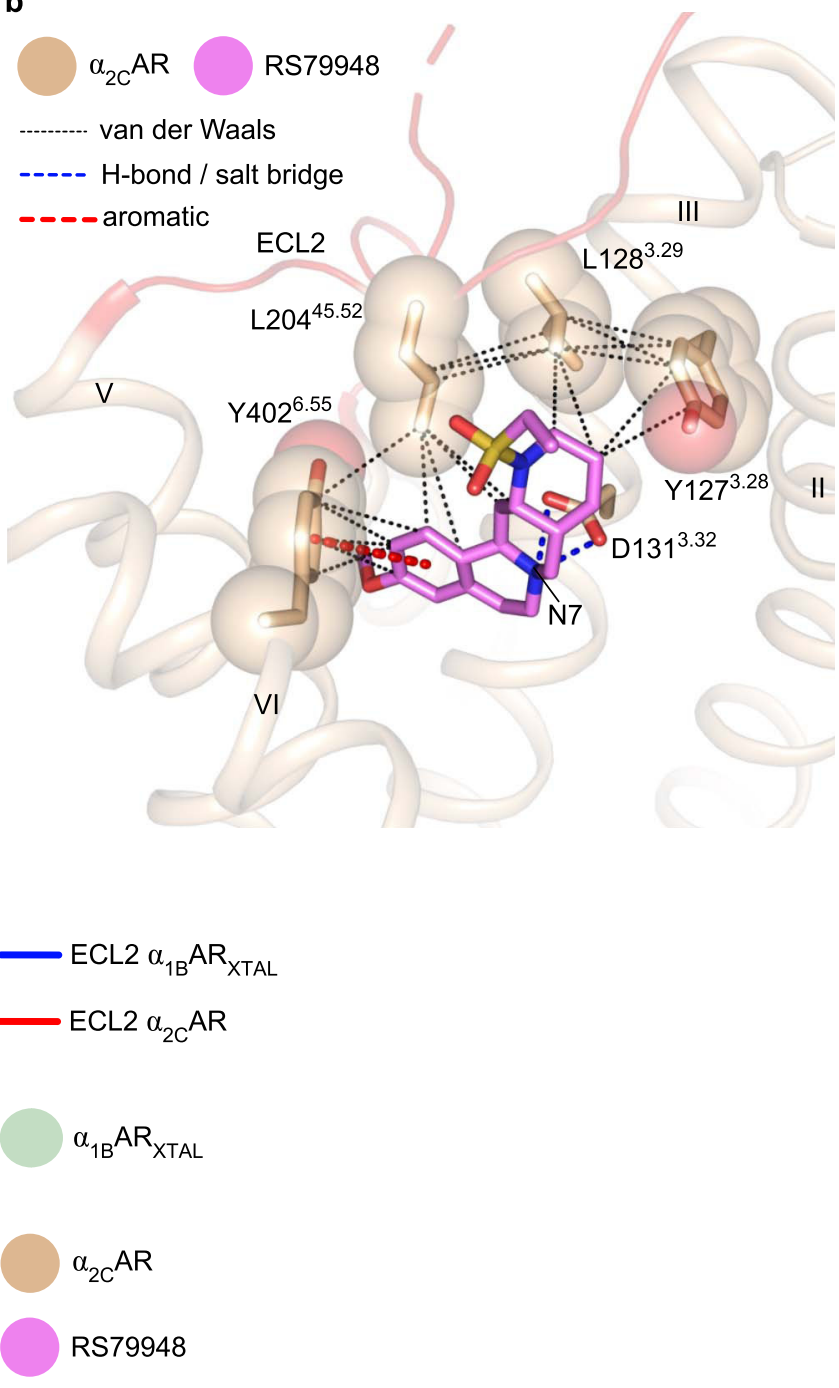

Fig. 4 Molecular determinants and structural basis for the selective binding of RS79948 to $\alpha_{\mathbf{2}} \mathbf{A R}$ over $\boldsymbol{\alpha}_{\mathbf{1 B}} \mathbf{A R}$. a Affinity of RS79948 for $\alpha_{1 B} A R$, $\alpha_{2 C} A R$, and chimeric $\alpha_{1 B} A R-\alpha_{2 C}$ mutants. Single amino acid substitutions in $\alpha_{1 B} A R$ are indicated in the construct names and correspond to the $\alpha_{2 C} A R$ sequence at either one of positions $3.28,3.29,45.52$, or 6.55 . The $\alpha_{1 B} A R-\alpha_{2 C}(Y L L Y)$ chimera corresponds to the quadruple mutant. Data are shown as mean values \pm standard error of the mean (SEM) of 3-6 independent experiments performed in triplicate. The underlying data points are depicted as black diamonds, and the exact $n$, SEM, and 95\% confidence interval of the mean are reported in Supplementary Table 6. Differences in affinities were evaluated by a statistical test as detailed in Supplementary Table 7. b Structural role of Y1273.28, L1283.29, L20445.52, and Y4026.55 in the binding of RS79948 to $\alpha_{2 C}$ AR (PDB ID: 6KUW26). TM1, ECL3, and TM7 have been omitted for clarity. Receptor residues are shown as van der Waals spheres and as sticks, except for D1313.32, which is shown as sticks only; RS79948 is shown as sticks. Oxygen, nitrogen, and sulfur atoms are depicted in red, blue, and yellow, respectively. c Superposition of $\alpha_{1 B} A R_{X T A L}$ bound to ( + )-cyclazosin and $\alpha_{2 C} A R-R S 79948$ (PDB ID: 6KUW26), focusing on the residues outlined in panel $b$. (+)-Cyclazosin, TM1, TM2, ECL1, ECL3, and TM7, have been omitted for clarity. Receptor residues and RS79948 are shown as sticks. V197 is shown to C $\beta$ only because its side chain is not resolved in the electron density map. Source data are provided as a Source Data file.

On the opposite side of the RS79948-binding pocket in $\alpha_{2 \mathrm{C}} \mathrm{AR}$, Y $402^{6.55}$ establishes aromatic and van der Waals interactions with the benzene moiety of this antagonist, probably restricting its degrees of freedom (Fig. 4b). The corresponding L314 ${ }^{6.55}$ residue in $\alpha_{1 \mathrm{~B}} \mathrm{AR}$, owing to its aliphatic side chain and smaller size, may form weaker interactions and allow more flexibility of this ligand (Figs. $3 \mathrm{~b}$ and $4 \mathrm{c}$ ). Due to the constrained polycyclic ring structure of RS79948, mispositioning of the benzene moiety could again negatively affect the interaction between the ligand's N7 atom and the side chain of D131 3.32 .

Finally, in addition to modulating RS79948 selectivity, the different properties of $\mathrm{A}^{3.29}$ in $\alpha_{1}$ ARs compared to $\mathrm{L}^{3.29}$ in $\alpha_{2} \mathrm{ARs}$ may underlie the distinct selectivity profiles of yohimbine and corynanthine (also known as rauhimbine), two RS79948-related compounds (Supplementary Fig. 11a). Although yohimbine and corynanthine differ in the configuration of only a single stereocenter (C5), yohimbine is a potent $\alpha_{2} \mathrm{AR}$-selective antagonist, while corynanthine has a substantially reduced affinity for $a_{2} \mathrm{ARs}^{54-56}$. Docking of these ligands to the $\alpha_{2 \mathrm{C}} \mathrm{AR}$ structure (PDB ID: $6 \mathrm{KUW}^{26}$ ) and to a model of $\alpha_{2 \mathrm{C}} \mathrm{AR}-\mathrm{L} 128^{3.29} \rightarrow \mathrm{A}$, together with $\mathrm{MD}$ simulations, suggests that binding of corynanthine is hampered by steric hindrance between its bulky methyl ester substituent and $\mathrm{L}^{3.29}$ in $\alpha_{2}$ ARs (Supplementary Fig. 11b-g and Supplementary Movie 1). In contrast, the same methyl ester substituent in yohimbine points away from $\mathrm{L}^{3.29}$, owing to the opposite stereochemical configuration at $\mathrm{C} 5$, 
a

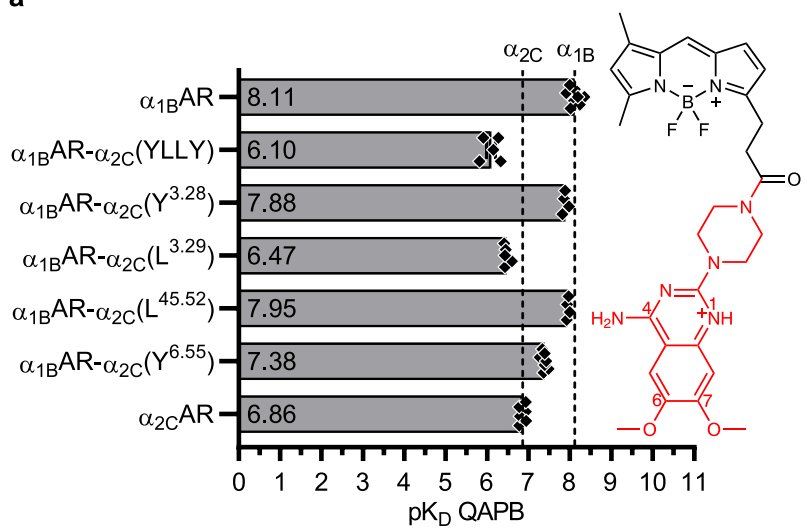

b

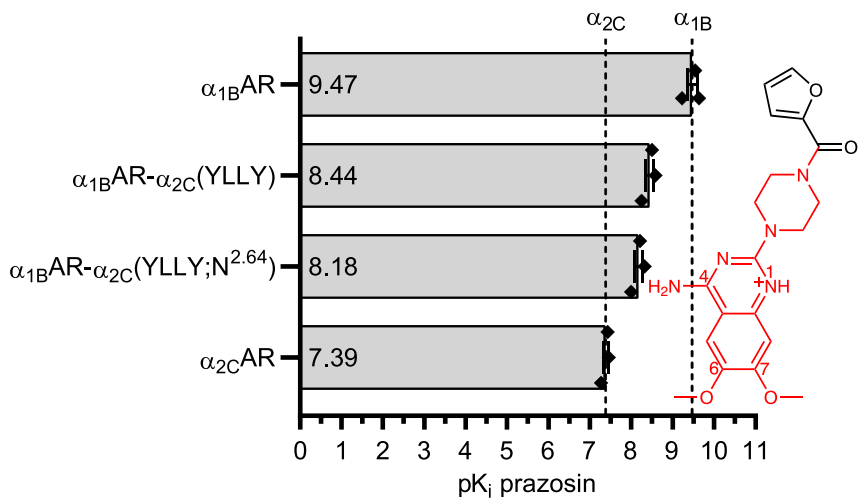

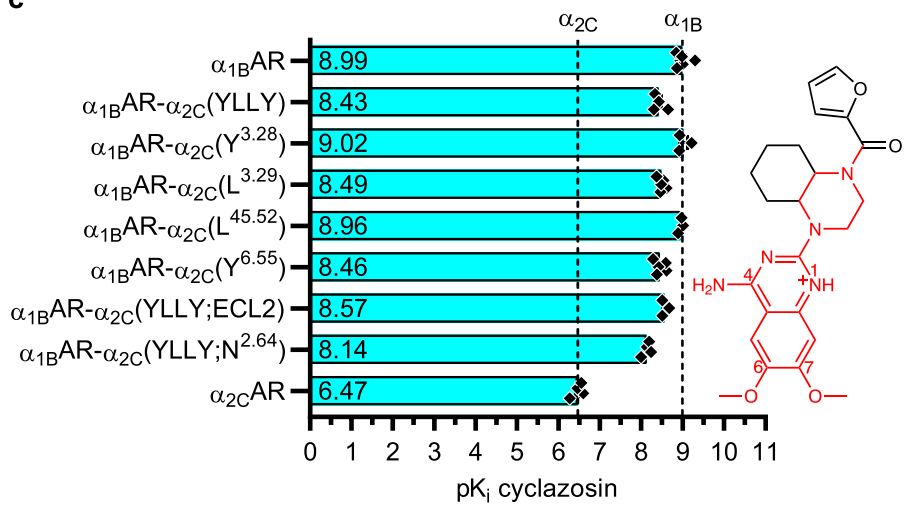

d

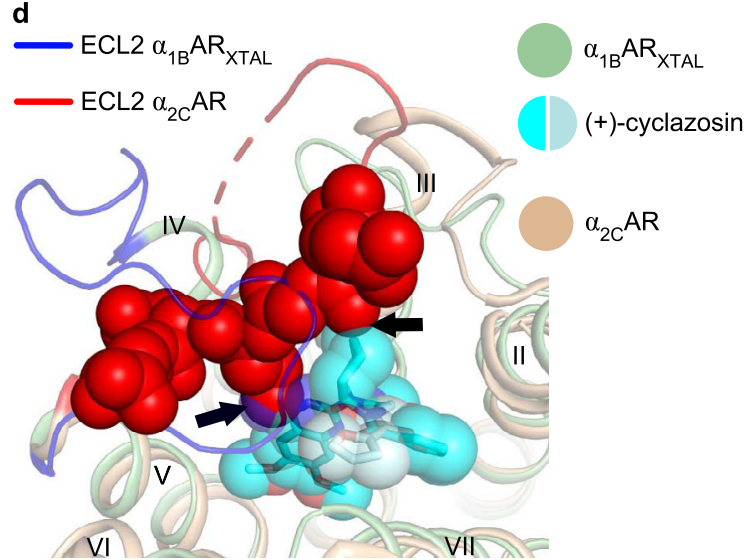

Fig. 5 Molecular determinants for the preferred binding of piperazinyl quinazolines to $\boldsymbol{\alpha}_{\mathbf{1}}$ ARs over $\boldsymbol{\alpha}_{\mathbf{2}}$ ARs. a-c Affinity of (a) QAPB, (b) prazosin, and (c) cyclazosin for $\alpha_{1 B} A R, \alpha_{2 C} A R$, and chimeric $\alpha_{1 B} A R-\alpha_{2 C}$ mutants. Single amino acid substitutions in $\alpha_{1 B} A R$ are indicated in the construct names and correspond to the $\alpha_{2 C} A R$ sequence at either one of positions 3.28, 3.29, 45.52, or 6.55. The $\alpha_{1 B} A R-\alpha_{2 C}(Y L L Y)$ chimera corresponds to the quadruple mutant. All three ligands share a common piperazinyl 4-amino-6,7-dimethoxyquinazoline scaffold, which is highlighted in red in their chemical structures. Data are shown as mean values \pm SEM of 3-6 independent experiments performed in triplicate. The underlying data points are depicted as black diamonds, and the exact $n$, SEM, and 95\% confidence interval of the mean are reported in Supplementary Table 6. Differences in affinities were evaluated by a statistical test as detailed in Supplementary Table 7. d Superposition of $\alpha_{1 B} A R_{X T A L}$ bound to (+)-cyclazosin and $\alpha_{2 C}$ AR-RS79948 (PDB ID: 6KUW26), focusing on the potential role of ECL2 in selective ligand binding. The ECL2 residues in $\alpha_{2 C} A R$ that form a lid on the ligand-binding site are shown as red van der Waals spheres. (+)-Cyclazosin is depicted as sticks and as transparent van der Waals spheres, with the two orientations observed for its furan-2-ylmethanone substituent colored in cyan and pale cyan, respectively. Black arrows indicate potential hindrance exerted by ECL2 with respect to $(+)$-cyclazosin. Source data are provided as a Source Data file.

averting steric hindrance with $\mathrm{L}^{3.29}$. Less hindrance is expected for binding of corynanthine to $\alpha_{1}$ ARs, since $\alpha_{1}$ ARs bear an alanine at position 3.29.

Molecular determinants of preferred ligand binding to $\alpha_{1} \mathrm{ARs}$ over $\boldsymbol{\alpha}_{2}$ ARs. To gain further understanding of selective ligand recognition within $\alpha A R s$, we investigated the preferential binding of piperazinyl 4-amino-6,7-dimethoxyquinazoline compounds to $\alpha_{1}$ ARs over $\alpha_{2} A_{R s}{ }^{57}$. Three such compounds, i.e., QAPB (quinazolinyl piperazine BODIPY), prazosin, and cyclazosin, displayed $\sim 20-, 120-$, and 330-fold higher affinity for wild-type $\alpha_{1 B} A R$ compared to $\alpha_{2 C} A R$, respectively (Fig. 5a-c, Supplementary Fig. 10, and Supplementary Table 6). The measured affinities agree well with previously reported values ${ }^{15,18}$. The $\alpha_{1 B} A R-$ $a_{2 C}$ (YLLY) chimera described above had $\sim 100-, 11-$, and 4 -fold weaker affinity for QAPB, prazosin, and cyclazosin, respectively, compared to wild-type $\alpha_{1 \mathrm{~B}} \mathrm{AR}$ (Fig. 5a-c). When assessed individually, the chimeric substitutions $\mathrm{A} 122^{3.29} \rightarrow \mathrm{L}$ and $\mathrm{L} 314^{6.55} \rightarrow \mathrm{Y}$ led to a 45- and 5-fold loss of QAPB affinity at $\alpha_{1 \mathrm{~B}} \mathrm{AR}$, respectively. These two substitutions also lowered cyclazosin affinity by $\sim 3$-fold each, whereas the effect of $\mathrm{W} 121^{3.28} \rightarrow \mathrm{Y}$ and $\mathrm{V} 197^{45.52} \rightarrow \mathrm{L}$ was negligible for both QAPB and cyclazosin. Based on these trends, it is conceivable that the higher steric bulk of $\mathrm{L}^{3.29}$ and $\mathrm{Y}^{6.55}$ in $\alpha_{2} \mathrm{ARs}$, compared to $\mathrm{A}^{3.29}$ and $\mathrm{L} / \mathrm{M}^{6.55}$ in $a_{1}$ ARs, hinders an optimal positioning of these piperazinyl quinazoline ligands in the binding pocket.

To assess the contribution of hydrophobic contacts mediated by the furan ring of prazosin and cyclazosin with TM2 (Fig. 2a, c) to the selectivity profile, we introduced the chimeric substitution $\mathrm{L} 105^{2.64} \rightarrow \mathrm{N}$, generating $\alpha_{1 \mathrm{~B}} \mathrm{AR}-\alpha_{2} \mathrm{C}\left(\mathrm{YLLY} ; \mathrm{N}^{2.64}\right)$. The affinity of prazosin and cyclazosin for this chimera appeared to decrease by $\sim 2$-fold compared to $a_{1 B} A R-a_{2 C}$ (YLLY) (Fig. 5b, c), however, this loss of affinity did not reach statistical significance (Supplementary Table 7). No further loss of cyclazosin affinity was observed for chimeric substitutions at the remaining two non-conserved positions (S208 ${ }^{5.43} \rightarrow \mathrm{C}$ and $\mathrm{D} 327^{7.32} \rightarrow \mathrm{G}$ ) within the binding site of $\alpha_{1 \mathrm{~B}} \mathrm{AR}$ (Supplementary Table 6).

We hypothesized that ECL2 of $\alpha_{2}$ ARs may play a role in the selectivity of piperazinyl 4-amino-6,7-dimethoxyquinazoline compounds for $a_{1}$ ARs over $a_{2}$ ARs. Thus, we measured the affinity of cyclazosin for the $\alpha_{1 B} A R-\alpha_{2 C}$ (YLLY;ECL2) chimera described above. No change in cyclazosin affinity was observed at $a_{1 B} A R-a_{2 C}$ (YLLY;ECL2) compared to $\alpha_{1 B} A R-a_{2 C}$ (YLLY) (Fig. 5c). Nonetheless, superposition of the $(+)$-cyclazosin complex with 
the structures of $\alpha_{2 \mathrm{~A}} \mathrm{AR}$ and $\alpha_{2 \mathrm{C}} \mathrm{AR}$ bound to RS79948 suggests that ECL2 hinders binding of piperazinyl 4-amino-quinazoline ligands to $\alpha_{2} \mathrm{ARs}$, in particular those with a constrained bulky group fused to the piperazine ring, such as cyclazosin (Fig. $5 \mathrm{~d}$ and Supplementary Fig. 7a, b). It is possible, however, that the $\alpha_{1 \mathrm{~B}} \mathrm{AR}-$ $a_{2 C}$ (YLLY;ECL2) chimera could not reproduce the effect of ECL2 on ligand selectivity because the conformation of this loop in $\alpha_{2}$ ARs is influenced by contacts with other receptor regions, as previously described ${ }^{26}$. The fact that the conformation of ECL2 in $\alpha_{2 \mathrm{~A}} \mathrm{AR}$ has already been shown to hinder binding of bulky ligands $^{26}$ is consistent with our postulated mechanism of selectivity.

Overall, we identified positions 3.29 and 6.55 as contributors to the $\alpha_{1} \mathrm{AR}$ selectivity of piperazinyl quinazoline compounds. Nonetheless, the molecular basis for the $\alpha_{1} A R$ selectivity of prazosin and cyclazosin could not yet be fully replicated from chimeras. We thus postulate that ECL2 in $\alpha_{2}$ ARs may sterically interfere with the binding of these ligands, in particular of cyclazosin, and thus contributes to the selectivity of these ligands for $a_{1}$ ARs.

\section{Discussion}

The emerging key roles of aARs in many physiological and pathophysiological processes, especially in the heart, CNS, and immune system $^{6-11,14}$, have suggested new avenues to treat cardiovascular, neurological, neuropsychiatric, and inflammatory disorders. Clearly, selective targeting of individual aAR subtypes will be crucial to fully exploit these receptors as drug targets. Moreover, due to the complexity of CNS disorders, their therapy often requires polypharmacology (i.e., interaction with multiple targets), which needs to be perfectly tailored to avoid severe off-target side effects.

A particular challenge is the high conservation of key ligandanchoring residues in the binding pockets of aminergic receptors ${ }^{16,17}$. For instance, the antipsychotics risperidone and haloperidol bind to the D2 dopamine receptor (DRD2) $)^{20,22}$ through contacting residues that are nearly entirely conserved in $\alpha_{1}$ ARs (Supplementary Fig. 12), explaining some of the off-target activity of these drugs ${ }^{18}$. To guide the drug discovery processeven on other targets-it is crucial to understand the molecular determinants underlying ligand selectivity or promiscuity at aARs.

The lack of structures for $\alpha_{1}$ ARs had largely precluded rational ligand design so far, and fully $\alpha_{1 \mathrm{~B}} \mathrm{AR}$-selective compounds are not available yet. To bridge this gap, we determined the crystal structure of human $\alpha_{1 \mathrm{~B}} \mathrm{AR}$. To enable crystallization, we had to introduce the receptor modifications typically required for crystallization of $\mathrm{GPCRs}^{58}$, i.e., stabilizing mutations, fusion of a crystallization chaperone, and truncation of long flexible regions. We stabilized the receptor in an inactive state using CHESS-based directed evolution ${ }^{42}$ and fused it to the DARPin D12 crystallization chaperone $\mathrm{e}^{43}$. The same strategy has recently allowed us to determine structures for several ligand complexes of NTSR $1^{44}$, which had been recalcitrant to crystallization before. Previously reported affinity data for the inverse agonist prazosin indicate that ligand binding to $\alpha_{1 \mathrm{~B}} \mathrm{AR}$ expressed in $E$. coli is not different from $\alpha_{1 \mathrm{~B}} \mathrm{AR}$ produced in mammalian cells ${ }^{15,59}$. In addition, we have recently shown that the ligand-binding site of the neurotensin receptor 1 (NTSR1) is virtually identical in structures obtained from bacterial or eukaryotic expression systems ${ }^{44}$. Although our study focuses on inactive-state $\alpha_{1 B} A R$, we note that the ICL3 deletion and the replacement of helix 8 by the DARPin fusion are likely to impair coupling to intracellular signaling proteins.

Alongside the previously reported $\beta \mathrm{AR}$ and $\alpha_{2} \mathrm{AR}$ structures, the complex of $\alpha_{1 \mathrm{~B}} \mathrm{AR}$ with (+)-cyclazosin now offers a comprehensive view of ligand binding at this clinically important subfamily of GPCRs. Previous studies had identified the nonconserved residue $\mathrm{F} / \mathrm{N}^{7.39}$ as a crucial determinant of $\beta A R$ versus aAR selectivity ${ }^{17,60}$. Our findings now reveal that residues $A / \mathrm{L}^{3.29}$ and $\mathrm{L} / \mathrm{Y}^{6.55}$ are important determinants of selectivity between $\alpha_{1}$ ARs and $\alpha_{2}$ ARs. Among aminergic receptors, $A^{3.29}$ is unique to $\alpha_{1}$ ARs. $L^{6.55}$ is unique to $\alpha_{1 B} A R$ and $\alpha_{1 D} A R$, while $M^{6.55}$ as in $\alpha_{1 A} A R$ is only found in the histamine $\mathrm{H} 3$ receptor (HRH3) ${ }^{16}$. Thus, both positions could now be exploited to design selective compounds devoid of unwanted side effects.

In aminergic GPCRs, residue 3.29 delineates the boundary between the orthosteric binding site and secondary binding pockets (the latter are also described as "extended", "minor", or "allosteric" binding sites in other studies). Due to its gatekeeper position, residue 3.29 can be expected to mediate ligand selectivity in other aminergic GPCRs, as sequence divergence is observed at the level of aminergic subfamilies, their subgroups, and even between certain subtypes within the same subgroup ${ }^{16}$. Indeed, in DRD2 and DRD4, the non-conserved residue at position 3.29 contributes to ligand selectivity ${ }^{61}$. In the muscarinic acetylcholine receptors, $\mathrm{W}^{3.28} \rightarrow \mathrm{A}$ and $\mathrm{L}^{3.29} \rightarrow \mathrm{A}$ differentially affect the binding of several orthosteric ligands, depending on their extension toward the extracellular side $\mathrm{e}^{24,62-65}$.

Characterization of unique secondary binding pockets for different receptor subtypes is a promising strategy for identifying subtype-selective ligands via structure-based approaches. Since there is great interest in obtaining compounds with exquisite selectivity for $\alpha_{1 B} A R$ over $\alpha_{1 A} A R$, we note that the $\alpha_{1 B} A R$ structure presented here suggests to leverage on the nonconserved cavity between TM3 and TM2 (L/F2.64). In analogy, in $\mathrm{DRD} 2$ and DRD4, sequence divergence in TM2 at and proximal to position 2.64 contributes to ligand selectivity, likely by steric effects ${ }^{61}$. Alternatively, one might take advantage of the $\alpha_{1 B} A R$ residue $A^{5.39}$, which is spatially proximal to position 6.55 and less bulky than $\mathrm{V}^{5.39}$ in $\alpha_{1 \mathrm{~A}} \mathrm{AR}$, to modulate the ligand's interaction with $\mathrm{D}^{3.32}$. The conformation of the C-terminal portion of ECL2, which partially caps the (+)-cyclazosin binding site, might deviate in the $\alpha_{1 \mathrm{~A}} \mathrm{AR}$ and $\alpha_{1 \mathrm{D}} \mathrm{AR}$ subtypes compared to $\alpha_{1 \mathrm{~B}} \mathrm{AR}$ due to non-conserved residues at the extracellular tip of TM5 $\left(\mathrm{A} / \mathrm{P}^{5.36}\right.$ and $\left.\mathrm{G} / \mathrm{F}^{5.37}\right)$ as well as within the loop itself. Sequence variation within ECL2 has been shown to be a source of subtype selectivity for some $\alpha$-adrenergic ligands ${ }^{26,48,66}$.

Finally, the binding mode of $(+)$-cyclazosin suggests a rationale for its inverse agonistic properties. The extension of this ligand toward the extracellular vestibule in the proximity of TM7 possibly prevents $\mathrm{F}^{7.39}$ from sealing the $\mathrm{OBS}$, which is triggered by agonistic ligands, and has been proposed to contribute to receptor activation ${ }^{35}$ (Supplementary Fig. 13).

In conclusion, this study presents the crystal structure of an $\alpha_{1} A R$, enabled by directed evolution and a recently established DARPin fusion-based crystallization design. We elucidated key molecular determinants of $\alpha_{1} \mathrm{AR}$ versus $\alpha_{2} \mathrm{AR}$ selectivity, improving our understanding of adrenergic GPCRs and providing new opportunities for structure-based ligand screening and rational drug design.

\section{Methods}

Directed evolution of $\alpha_{1 \mathbf{B}} \mathbf{A R}$. Error-prone PCR was applied to the enriched pool from a previously described library of $\alpha_{1 B} A R$ (termed EP2AS3) ${ }^{41}$. The resultant library, with a diversity of $\sim 300,000$ clones, was subjected to one round of bacterial display for high expression ${ }^{67}$ using the fluorescent ligand QAPB (quinazolinyl piperazine BODIPY, also termed BODIPY FL prazosin, ThermoFisher Scientific) From the 1\% most fluorescent (highest QAPB-bound) cell population, 100,000 cells were sorted by fluorescence-activated cell sorting (FACS), as previously described $^{41}$. This resultant population was subsequently subjected to two rounds of CHESS (sorting of encapsulated E. coli for detergent stability of the receptor) ${ }^{42}$. The first round was carried out with solubilization in PBS-E $\left(10 \mathrm{mM} \mathrm{Na}_{2} \mathrm{HPO}_{4}\right.$,
} 
$1.8 \mathrm{mM} \mathrm{KH}_{2} \mathrm{PO}_{4}, 137 \mathrm{mM} \mathrm{NaCl}, 2.7 \mathrm{mM} \mathrm{KCl}, 1 \mathrm{mM}$ EDTA, $\mathrm{pH}$ 7.4) containing $2 \%(\mathrm{w} / \mathrm{v}) \mathrm{n}$-decyl- $\beta$-D-maltopyranoside (DM, Anatrace), $0.5 \%$ (w/v) CHAPS (Sigma-Aldrich), and $200 \mathrm{nM}$ QAPB, for $2 \mathrm{~h}$ at $4{ }^{\circ} \mathrm{C}$, followed by incubation in PBS-E containing $1 \%(w / v)$ 1,2-diheptanoyl-sn-glycero-phosphocholine $\left(\mathrm{DH}_{7} \mathrm{PC}\right.$, Anatrace) and $200 \mathrm{nM} \mathrm{QAPB}$, for $30 \mathrm{~min}$ at $4{ }^{\circ} \mathrm{C}$, and then PBS-E containing $0.33 \%$ (w/v) $\mathrm{DH}_{7} \mathrm{PC}$ and $200 \mathrm{nM}$ QAPB, for $24 \mathrm{~h}$ at $25^{\circ} \mathrm{C}$. Of the highest (top $1 \%$ ) QAPB fluorescent capsules, 26,000 capsules were sorted from the population using FACS, and the selected clones were isolated from the capsules with PCR, before being religated into the expression vector as described previously ${ }^{41}$. The second round of CHESS was applied with encapsulated cells solubilized as for the first round (see above), but with a final incubation in PBS-E containing $0.33 \%$ (w/v) $\mathrm{DH}_{7} \mathrm{PC}$ and $200 \mathrm{nM}$ QAPB, for $24 \mathrm{~h}$ at $37^{\circ} \mathrm{C}$. Of the highest (top 1\%) QAPB fluorescent capsules, 220,000 capsules were sorted, the clones isolated, re-ligated, and subjected to a final round of bacterial display, performed as above. Twenty-four selected $\alpha_{1 B} A R$ clones were screened individually as previously described ${ }^{41}$. Following characterization of the clones, the best-behaved clone was referred to as $\alpha_{1 B} \mathrm{AR}-\mathrm{B} 1$ and is described elsewhere ${ }^{59}$. Twelve of the 14 amino acid substitutions harbored by $a_{1 B} A R-B 1$ compared to wild-type human $\alpha_{1 B} A R$ were transferred to the crystallization construct (see below and Supplementary Table 1), as they were in receptor regions not subjected to truncations.

Generation of $\boldsymbol{\alpha}_{\mathbf{1 B}} \mathbf{A} \mathbf{R}_{\mathbf{X T A L}}$. The annotated amino acid sequence of $\alpha_{1 \mathrm{~B}} \mathrm{AR}_{\mathrm{XTAL}}$ is available in the PDB under the accession code 7B6W as well as in Supplementary Fig. $2 c$. Briefly, $\alpha_{1 B} A R_{X T A L}$ was obtained by introducing the following modifications in the sequence of wild-type human $a_{1 B} A R$ : introduction of 12 amino acid substitutions (Supplementary Table 1) derived from directed evolution (see above), deletion of N-terminal residues M1-N34 and intracellular loop three (ICL3) residues K249-L283, and fusion of DARPin D12 ${ }^{43,68,69}$ to residue C $350^{7.55}$ via the linker sequence AEDLVEDWE (Supplementary Fig. 2a, c). This sequence has previously been used to fuse DARPin D12 to the neurotensin receptor 1 $(\mathrm{NTSR} 1)^{44}$, resulting in a shared helix between TM7 of NTSR1 and the N-terminal region of DARPin D12; however, in the complex of $\alpha_{1 \mathrm{~B}} \mathrm{AR}_{\mathrm{XTAL}}$ with $(+)$-cyclazosin, this linker region lacked regular secondary structure (Supplementary Fig. 2b). DARPin D12 was modified in its $\mathrm{N}$-terminal region by deletion of residues $\mathrm{S} 1$ and D2, and by introduction of four point mutations, i.e., $\mathrm{L} 3 \rightarrow \mathrm{K}, \mathrm{G} 4 \rightarrow \mathrm{A}$, $\mathrm{K} 5 \rightarrow \mathrm{R}$ and $\mathrm{A} 13 \rightarrow \mathrm{K}$ (Supplementary Fig. 2a, c), as a result of sequence optimization with Rosetta fix $b b^{70}$. The aim was to design a fusion site between GPCR and DARPin featuring a compromise between rigidity and malleability in order to adapt to different crystal packings. Furthermore, the last two C-terminal DARPin D12 residues, L157 and N158, were both mutated to alanine. As depicted in Supplementary Fig. 2c, the DARPin is followed by a short linker (sequence: TRE), followed by a cleaved human rhinovirus (HRV) 3C protease cleavage site (sequence: LEVLFQ). This turned out to be partially a-helical and established crystal contacts (Supplementary Figs. 2b and 4d). For expression in E. coli, the gene encoding the receptor construct was cloned into a previously described pBR322derived vector ${ }^{71}$. Briefly, this resulted in an expression construct consisting of an $\mathrm{N}$-terminal maltose-binding protein (MBP), followed by a $\mathrm{His}_{6}$-tag, a HRV $3 \mathrm{C}$ protease cleavage site (sequence: LEVLFQGP), a short linker (sequence: GS), the receptor itself fused to the modified DARPin D12, a short linker (sequence: TRE), a second HRV 3C protease cleavage site (sequence: LEVLFQGP), followed by thioredoxin A (TrxA) and a C-terminal His $1_{10}$-tag. The HRV $3 \mathrm{C}$ protease cleaves the peptide bond between $\mathrm{Q}$ and $\mathrm{G}$ of the above-mentioned cleavage site.

Generation of the prazosin ligand-affinity column. The prazosin column consists of a maleimide-(PEG2) ${ }_{2}$-prazosin derivative (Supplementary Fig. 14) coupled to the unique C-terminal Cys added via a linker to protein $\mathrm{D}(\mathrm{pD}$-Cys), which is in turn coupled via amino groups to NHS-activated Sepharose beads. pD-Cys corresponds to the $\mathrm{pD}-\mathrm{NT}$ variant previously described ${ }^{71}$, with the difference that the C-terminal HRV 3C protease cleavage site and the NT8-13 epitope are replaced by the amino acid sequence GGGGSGGGC. Expression and purification of pD-Cys were carried out as described for $\mathrm{pD}-\mathrm{NT}^{71}$, with the difference that EDTA pH 8.0 was added to a final concentration of $10 \mathrm{mM}$ to the protein sample after elution from the Ni-NTA column, followed by protein concentration and dialysis as described $^{71}$. Subsequently, to protect the cysteine from coupling to the NHSactivated Sepharose (otherwise a side reaction we suspected to occur), the pD-Cys protein was dimerized via a disulfide bond. To this end, $\mathrm{CuCl}_{2}$ was added to a final concentration of $1 \mathrm{mM}$ and the mixture incubated overnight at $4{ }^{\circ} \mathrm{C}$, followed by $1 \mathrm{~h}$ at room temperature. Typically, $\sim 85 \%$ of $\mathrm{pD}$-Cys could be dimerized according to analytical gel filtration on an S200 5/150 GL column. Coupling of dimerized protein via its lysines and $\mathrm{N}$ terminus to NHS-activated Sepharose (GE Healthcare) was carried out as described ${ }^{71}$, followed by blocking with Tris, but the subsequent washing buffers were adjusted to contain $10 \mathrm{mM}$ EDTA pH 8.0. After washing with guanidine hydrochloride and $\mathrm{H}_{2} \mathrm{O}$ as described ${ }^{71}$, the disulfide bond was reduced by incubation with two column volumes (CV) of Reducing Buffer (50 mM HEPES $\mathrm{pH}$ 8.0, $25 \mathrm{mM}$ TCEP, $10 \mathrm{mM}$ EDTA $\mathrm{pH} 8.0$ ) for $30 \mathrm{~min}$ at room temperature. Afterward, the resin was washed with Coupling Buffer $(100 \mathrm{mM}$ Na phosphate $\mathrm{pH}$ 6.0 , degassed) and subsequently incubated with $1 \mathrm{CV}$ of $0.8 \mathrm{mM}$ maleimide(PEG2) ${ }_{2}$-prazosin (Anawa) in Coupling Buffer for $30 \mathrm{~min}$ at room temperature. This resulted in $\sim 0.5 \mathrm{nmol}$ ligand/ $\mu \mathrm{l}$ resin as measured by comparison of the prazosin absorption at $330 \mathrm{~nm}$ before and after coupling. The resulting prazosin affinity column was finally washed with Coupling Buffer and $\mathrm{H}_{2} \mathrm{O}$, and stored in $20 \% \mathrm{EtOH}$ at $4{ }^{\circ} \mathrm{C}$. According to our experience, the prazosin column can be used multiple times and is stable for years without any major loss in binding capacity.

Expression and purification of $\alpha_{1 \mathrm{~B}} \mathrm{AR}_{\mathbf{X T A L}} \cdot \alpha_{1 \mathrm{~B}} \mathrm{AR}_{\mathrm{XTAL}}$ was expressed in $E$. coli BL21 cells bearing a deletion of the fhuA2 gene to confer phage T1 resistance (New England Biolabs) as previously described for other stabilized $\alpha_{1 B} A R$ variants ${ }^{59}$. The resulting cell pellet was resuspended with Resuspension Buffer (100 mM HEPES $\mathrm{pH} 8.0,30 \%(\mathrm{v} / \mathrm{v})$ glycerol, $400 \mathrm{mM} \mathrm{NaCl})$ at $4{ }^{\circ} \mathrm{C}$, frozen in liquid nitrogen, and stored at $-80^{\circ} \mathrm{C}$. All the following steps were carried out at $4{ }^{\circ} \mathrm{C}$. Typically, $90 \mathrm{ml}$ of frozen resuspended cells (corresponding to $30 \mathrm{~g}$ of the pellet) were thawed, $45 \mathrm{ml}$ of $\mathrm{H}_{2} \mathrm{O}$ were added, and the resuspension was incubated with $2 \mathrm{mg} / \mathrm{ml}$ lysozyme (Sigma-Aldrich), $5 \mathrm{mM} \mathrm{MgCl}$, and $0.05 \mathrm{mg} / \mathrm{ml}$ DNase I (Roche) for $10 \mathrm{~min}$ while stirring. Subsequently, receptors were solubilized by incubation with $1.67 \%(\mathrm{w} / \mathrm{v})$ $\mathrm{n}$-dodecyl- $\beta$-D-maltopyranoside (DDM, Anatrace) and $0.33 \%(\mathrm{w} / \mathrm{v})$ cholesteryl hemisuccinate (CHS, Sigma-Aldrich) for $30 \mathrm{~min}$ while stirring followed by sonication for 15 min using a Sonifier 250 (Branson) at a duty cycle of $30 \%$ and output 5 . The lysate containing detergent-solubilized receptors was stirred for another $1 \mathrm{~h}$, then adjusted with imidazole $(\mathrm{pH} 8.0)$ to a final concentration of $15 \mathrm{mM}$ and centrifuged at $20,000 \times g$ for $30 \mathrm{~min}$. The supernatant was batch-incubated for $2.5 \mathrm{~h}$ with $30 \mathrm{ml}$ of TALON Superflow resin (GE Healthcare) equilibrated with TALON Wash Buffer I (TWB-I; $25 \mathrm{mM}$ HEPES pH 8.0, 10\% (v/v) glycerol, $600 \mathrm{mM} \mathrm{NaCl}$, $0.1 \%(w / v)$ DDM, $0.02 \%(w / v)$ CHS, $15 \mathrm{mM}$ imidazole $\mathrm{pH} 8.0$ ). Subsequently, the resin was washed with 18 column volumes (CV) of TWB-I followed by $18 \mathrm{CV}$ of TWB-II (25 mM HEPES pH 7.0, $10 \%$ (v/v) glycerol, $150 \mathrm{mM} \mathrm{NaCl}, 0.1 \%(\mathrm{w} / \mathrm{v})$ DDM, $0.02 \%(\mathrm{w} / \mathrm{v}) \mathrm{CHS})$. Protein elution was carried out with $3 \mathrm{CV}$ of TALON Elution Buffer (TEB; $25 \mathrm{mM}$ HEPES pH 8.0, $10 \%$ (v/v) glycerol, $150 \mathrm{mM} \mathrm{NaCl}$, $0.1 \%(\mathrm{w} / \mathrm{v})$ DDM, $0.02 \%(\mathrm{w} / \mathrm{v})$ CHS, $200 \mathrm{mM}$ EDTA pH 8.0).

The eluted protein was concentrated in six $100 \mathrm{kDa}$ molecular weight cutoff Vivaspin 20 concentrators (Sartorius) to a total volume of $\sim 30 \mathrm{ml}$. Subsequently, the protein was incubated for $2 \mathrm{~h}$ with HRV $3 \mathrm{C}$ protease (produced in-house) to cleave off the fusion proteins MBP and TrxA, after which $2 \mathrm{ml}$ of prazosin column (PC) resin equilibrated in TEB was added and incubated overnight while rolling in a Falcon tube. The resin was subsequently transferred into an empty PD-10 column, washed with 2 CV of TEB, four times with 3 CV of PCWB-I $(25 \mathrm{mM}$ HEPES pH 8.0, $10 \%$ (v/v) glycerol, $600 \mathrm{mM} \mathrm{NaCl}, 0.05 \%$ (w/v) DDM, $0.01 \%$ (w/v) CHS, $200 \mathrm{mM}$ EDTA pH 8.0), three times with 2 CV of PCWB-II (10 mM HEPES pH 7.5, $10 \%(\mathrm{v} / \mathrm{v})$ glycerol, $100 \mathrm{mM} \mathrm{NaCl}, 0.018 \%(\mathrm{w} / \mathrm{v}) \mathrm{DDM}, 0.0036 \%(\mathrm{w} / \mathrm{v})$ CHS), and partially equilibrated with $0.3 \mathrm{CV}$ of PCEB (10 mM HEPES pH 7.5, $10 \%(\mathrm{v} / \mathrm{v})$ glycerol, $100 \mathrm{mM} \mathrm{NaCl}, 0.018 \%(\mathrm{w} / \mathrm{v}) \mathrm{DDM}, 0.0036 \%(\mathrm{w} / \mathrm{v})$ CHS, $75 \mu \mathrm{M}$ cyclazosin). For elution of cyclazosin-bound receptor, $0.75 \mathrm{CV}$ of PCEB were added to the column and incubated while rolling for $2.5 \mathrm{~h}$. After the addition of an additional $30 \mu \mathrm{M}$ cyclazosin (Sigma-Aldrich), the eluant as well as a wash with $0.5 \mathrm{CV}$ of PCEB were collected, resulting in an elution volume of 2-2.5 ml. In order to determine the protein concentration, excess cyclazosin had to be removed. To this end, an analytical fraction of the sample was loaded onto a Zeba Spin desalting column ( $7 \mathrm{kDa}$ molecular weight cutoff, ThermoFisher Scientific) equilibrated with PCWB-II. Absorption at $280 \mathrm{~nm}$ was measured using a Nanodrop 2000 spectrophotometer (ThermoFisher Scientific) and it was corrected by multiplication with an empirically determined correction factor of 0.7 to take into account absorption of receptor-bound cyclazosin. A suitable fraction of the protein sample was then concentrated with a $100 \mathrm{kDa}$ molecular weight cutoff Vivaspin 2 concentrator (Sartorius) to $\sim 50 \mathrm{mg} / \mathrm{ml}$, resulting in a typical final volume of $25 \mu \mathrm{l}$.

\section{Crystallization of $\alpha_{1 \mathrm{~B}} \mathbf{A} \mathbf{R}_{\mathbf{X T A L}}$ in the lipidic cubic phase. Cyclazosin-bound} $\alpha_{1 B} A R_{X T A L}$ was reconstituted in lipidic cubic phase (LCP) by mixing concentrated protein $(\sim 50 \mathrm{mg} / \mathrm{ml})$ with molten monoolein (Sigma-Aldrich) supplemented with $10 \%(\mathrm{w} / \mathrm{w})$ cholesterol (Sigma-Aldrich) at a protein-to-lipid ratio of 20:31 (v/v) using the two-syringe method (100- $\mu \mathrm{l}$ syringes, Hamilton). Crystallization trials were carried out at $20^{\circ} \mathrm{C}$ in 96 -well glass sandwich plates (SWISSCI) with a 120 $\mu \mathrm{m}$ spacer. A Crystal Gryphon LCP crystallization robot (Art Robbins Instruments) was used to dispense either $25 \mathrm{nl}$ or $40 \mathrm{nl}$ boli and to cover them with $800 \mathrm{nl}$ of precipitant solution. The plates were immediately sealed with a cover glass and incubated at $20^{\circ} \mathrm{C}$ in a Rock Imager 1000 (Formulatrix). The crystals obtained in this study were of rather small size, typically not exceeding $\sim 15-30 \mu \mathrm{m}$ in any dimension. Optimized crystallization conditions consisted of $100 \mathrm{mM}$ MES pH 6.0, 400-480 mM Li citrate, 28-33\% (w/v) PEG400, 10 mM L-glutathione reduced form, $10 \mu \mathrm{M}$ cyclazosin. $25 \mathrm{nl} \mathrm{LCP}$ boli tended to yield better-diffracting crystals. Crystals were harvested by picking the entire bolus at room temperature with a 25 $\mu \mathrm{m}$ MicroMesh (MiTeGen) and flash-frozen in liquid nitrogen without adding further cryoprotectant.

Data collection, structure determination, and structural analysis. X-ray diffraction data were collected from frozen LCP crystals at the X06SA (PXI) beamline at the Swiss Light Source (SLS) of the Paul Scherrer Institute (PSI, Villigen, Switzerland) on an EIGER 16M detector. Promising crystals were identified using a grid-scan protocol implemented in the SSX suite ${ }^{72}$. From these crystals, 109 partial datasets (minisets) were recorded, which were then indexed and integrated with 
$X D S^{73}$. Twenty-seven minisets, ranging between $15^{\circ}$ and $32^{\circ}$ wedge angle, were selected for the similarity in their cell parameters (to exclude possibly occurring non-isomorphous crystals) and were scaled with AIMLESS ${ }^{74}$ within the CCP4 package ${ }^{75}$. As a deltaB value of $17 \AA^{2}$ in AIMLESS $^{74}$ indicated moderate anisotropy, the scaled data were anisotropy-corrected and merged using the STARANISO server ${ }^{46}$ from Global Phasing Ltd. The structure of $\alpha_{1 B} \mathrm{AR}_{\mathrm{XTAL}}$ was determined by molecular replacement with the help of $P H A S E R^{76}$ using the coordinates of turkey $\beta_{1} \mathrm{AR}$ (PDB ID: 6IBL) and DARPin 5m3_D12 (PDB ID: 5LW2) as the search model, respectively. The single solution from $P H A S E R^{76}$ was refined by multiple rounds of model building in $\mathrm{COOT}^{77}$ and maximum likelihood refinement with $B U S T E R^{78}$ and $R E F M A C^{79}$. Validation during the course of refinement was performed using MolProbity ${ }^{80}$. Statistics for data collection and refinement can be found in Supplementary Table 3. A summary of the geometrical quality of the model is reported here below.

The overall MolProbity ${ }^{80}$ score is 1.76 and the all-atom clashscore is 5. In addition, $94.2 \%$ of the residues are within Ramachandran favored regions and 5.6\% within allowed regions. There is only one Ramachandran plot outlier (D357) in the unstructured linker between GPCR and DARPin crystallization chaperone. Finally, there are four side-chain rotamer outliers (F202, Y223, F284, and Q524, i.e., only $\sim 1 \%$ of the analyzed side chains). There are no such rotamer outliers in the ligandbinding site; one outlier is in the crystallization chaperone (Q524), while two of the remaining three outliers are immediately preceding or following an unstructured region (F202 and F284).

Receptors were structurally aligned using the command "align" in PyMOL (version 2.4.0a0), allowing five cycles of refinement on all atoms or on backbone atoms unless otherwise stated. For the $\alpha_{2 C}$ AR-RS79948 structure (PDB ID: $6 \mathrm{KUW}^{26}$ ), the coordinates of chain A were used for structural analysis. The OBS in the $\beta_{2} \mathrm{AR}$-epinephrine complex consists of residues $3.32,3.33,3.36,5.42,5.46,6.48$, $6.51,6.52,6.55,7.39$, and $7.43^{16}$

Mammalian cell culture. HEK293T/17 cells (American Type Culture Collection) were maintained in Dulbecco's modified medium (Sigma) supplemented with 100 units/ml penicillin, $100 \mu \mathrm{g} / \mathrm{ml}$ streptomycin (Sigma), and $10 \%$ (v/v) fetal calf serum (BioConcept). Cells were maintained at $37^{\circ} \mathrm{C}$ in a humidified atmosphere of $5 \%$ $\mathrm{CO}_{2}, 95 \%$ air.

Ligand-binding assays. Ligand-binding experiments were performed on transiently transfected HEK293T/17 cells using a homogeneous time-resolved fluorescence (HTRF) binding assay. All receptor variants were cloned into a mammalian expression vector containing an $\mathrm{N}$-terminal SNAP tag (Cisbio). The construct $\alpha_{1 \mathrm{~B}} \mathrm{AR}_{\mathrm{XTAL}}$ used for ligand-binding experiments is otherwise identical to the one used for crystallization (see above), including a cleaved 3C protease site (GP) followed by a short linker (GS) at the $\mathrm{N}$ terminus, and a short linker (TRE) followed by a cleaved $3 \mathrm{C}$ protease site (LEVLFQ) at the $\mathrm{C}$ terminus. This design also applies to the construct $\alpha_{1 B} A_{R_{X T A L}}-\Delta D 12$. However, in this construct, DARPin D12 was not fused to TM7 of the receptor, and the receptor was instead truncated at G369 after helix 8. Wild-type $\alpha_{1 \mathrm{~B}} \mathrm{AR}$ and the chimeric $\alpha_{1 \mathrm{~B}} \mathrm{AR}-\alpha_{2 \mathrm{C}}$ mutants were $\mathrm{N}$-terminally truncated to start at residue S35 to provide spatial proximity between the SNAP tag and the fluorescent ligand used as the tracer. Analogously, wild-type $a_{2 \mathrm{C}} \mathrm{AR}$ was $\mathrm{N}$-terminally truncated to start at residue A37. The wild-type receptors and the chimeric $\alpha_{1 B} A R-\alpha_{2 C}$ mutants harbored the wild-type full-length $C$ terminus.

For the HTRF ligand-binding assay, the receptors were labeled by covalently linking a Lumi4-Tb fluorophore (Cisbio) to the N-terminal SNAP tag of the receptor. Lumi4-Tb was used as a FRET (Förster resonance energy transfer)-donor (excitation at $340 \mathrm{~nm}$ and emission at $490 \mathrm{~nm}$ and $620 \mathrm{~nm}$ ). The $\alpha_{1}$ AR ligand QAPB was used as a FRET-acceptor (excitation at around $490 \mathrm{~nm}$ and emission at $520 \mathrm{~nm}$ ). If donor and acceptor are in spatial proximity and the donor is excited at $340 \mathrm{~nm}$, the donor's emission at $490 \mathrm{~nm}$ is quenched by the acceptor, whose emission at $520 \mathrm{~nm}$ is increased. This setup enabled the simultaneous measurement of receptor bound to the ligand QAPB (emission intensity at $520 \mathrm{~nm}$ ) and total receptor (emission intensity at $620 \mathrm{~nm}$ ). Both together allowed us to normalize binding to the receptor expression levels as well as to determine the relative receptor expression levels in our cell samples.

HEK293T/17 cells were harvested by trypsinization. Cells were reversetransfected with a mix of $863 \mathrm{ng} / \mathrm{ml}$ DNA and $15.43 \mu \mathrm{l} / \mathrm{ml}$ TransIT-293T ${ }^{\oplus}$ (Mirus Bio) in Opti-MEM (Gibco), which was incubated for $20-30 \mathrm{~min}$ at room temperature and subsequently $471.6 \mu \mathrm{l}$ thereof supplemented to $6 \mathrm{ml}$ of a cell resuspension containing $\sim 185,000 \mathrm{cells} / \mathrm{ml}$. This cell mix was dispensed to poly-Dlysine (Gibco)-coated 384-well plates (Greiner 781080) with $40 \mu \mathrm{l} /$ well and incubated $46-50 \mathrm{~h}$ at $37^{\circ} \mathrm{C}$ and $5 \% \mathrm{CO}_{2}$. Medium was discarded, $15 \mu \mathrm{l} /$ well $50 \mathrm{nM}$ SNAP-Lumi4-Tb (Cisbio) in assay buffer ( $20 \mathrm{mM}$ HEPES pH 7.5, $100 \mathrm{mM} \mathrm{NaCl}$, $3 \mathrm{mM} \mathrm{MgCl} 2,0.05 \%$ (w/v) BSA, $0.2 \%$ (w/v) skim milk) were added, and the plate was incubated at $37^{\circ} \mathrm{C}$ for $1.5 \mathrm{~h}$. The solution was discarded, and the cells were washed four times with $50 \mu \mathrm{l} /$ well ice-cold assay buffer. For saturation ligandbinding experiments, labeled cells were incubated with a dilution series of QAPB (ThermoFisher Scientific) in assay buffer ranging from $1 \times 10^{-6} \mathrm{M}$ to $2.92 \times 10^{-11} \mathrm{M}$. For competition ligand-binding experiments, labeled cells were incubated with a serial dilution of cyclazosin (Sigma-Aldrich), RS79948 (Tocris), or prazosin (Tocris) prepared in assay buffer, in the presence of $2 \mathrm{nM}$ QAPB for constructs $\alpha_{1 B} A R, \alpha_{1 B} A R-\alpha_{2 C}\left(Y^{3.28}\right), \alpha_{1 B} A R-\alpha_{2 C}\left(L^{45.52}\right), \alpha_{1 B} A R-\alpha_{2 C}\left(Y^{6.55}\right), \alpha_{1 B} A R-$ $\alpha_{2 C}\left(Y_{L L Y} ; N^{2.64} ; \mathrm{G}^{7.32}\right), \alpha_{1 B} A R-\alpha_{2 C}\left(Y_{L L Y} ; N^{2.64} ; C^{5.43}\right), \alpha_{1 B} A R_{X T A L}$, and $\alpha_{1 B} A R_{X T A L}$ $\triangle \mathrm{D} 12$, or $50 \mathrm{nM}$ QAPB for $\alpha_{2 \mathrm{C}} \mathrm{AR}, \alpha_{1 \mathrm{~B}} \mathrm{AR}-\mathrm{a}_{2 \mathrm{C}}\left(\mathrm{L}^{3.29}\right), \alpha_{1 \mathrm{~B}} \mathrm{AR}-\alpha_{2 \mathrm{C}}$ (YLLY), $\alpha_{1 \mathrm{~B}} \mathrm{AR}-$ $\alpha_{2 C}\left(\right.$ YLLY;ECL2), and $\alpha_{1 B} A R-\alpha_{2 C}\left(Y L L Y ; N^{2.64}\right)$. After incubation on ice for $2-4 \mathrm{~h}$, emission intensities at $520 \mathrm{~nm}$ and $620 \mathrm{~nm}$ after an excitation at $340 \mathrm{~nm}$ were measured on a SPARK fluorescence plate reader (Tecan). The ratio of emission intensities of FRET-acceptor and FRET-donor (Em520 nm/Em620 nm) was calculated, and $\mathrm{K}_{\mathrm{D}}$ and $\mathrm{IC}_{50}$ values were obtained by fitting the data with a fourparameter non-linear regression with GraphPad Prism Suite 8.4.3. $K_{\mathrm{i}}$ values were calculated using the Cheng-Prusoff equation ${ }^{81}$.

Signaling assays. Signaling assays were performed with receptor constructs harboring the wild-type helix 8 and the full-length C-terminus as well as the entire ICL3. Agonist-induced IP1 accumulation was measured in transiently transfected HEK293T/17 cells, as described before ${ }^{82}$. Twenty-four hours after transfection, cells were washed with phosphate-buffered saline (PBS), detached with trypsin-EDTA (Sigma), and resuspended in assay buffer (10 mM HEPES pH 7.4, $1 \mathrm{mM} \mathrm{CaCl}, 0.5 \mathrm{mM} \mathrm{MgCl}, 4.2 \mathrm{mM} \mathrm{KCl}, 146 \mathrm{mM} \mathrm{NaCl}, 50 \mathrm{mM} \mathrm{LiCl}, 5.5$ $\mathrm{mM}$ glucose, $0.1 \%(\mathrm{w} / \mathrm{v}) \mathrm{BSA})$. Cells were seeded at 20,000 cells per well in white 384-well plates (Greiner) and incubated for $2 \mathrm{~h}$ at $37^{\circ} \mathrm{C}$ with a concentration range of phenylephrine (Tocris) diluted in assay buffer. IP1 accumulation was measured using the HTRF IP-One kit (Cisbio) according to the manufacturer's protocol on a SPARK fluorescence plate reader (Tecan). This kit uses an anti-IP1 antibody labeled with Tb-cryptate as FRET-donor (excitation at $317 \mathrm{~nm}$, emission at $620 \mathrm{~nm}$ ) and IP1-d2 as FRET-acceptor (emission at $655 \mathrm{~nm}$ ). The ratio of emission intensities of FRET-acceptor and FRET-donor (Em655 nm/Em620 nm) was calculated, and $\mathrm{EC}_{50}$ (median effective concentration) values were obtained by fitting the data with a three-parameter non-linear regression with GraphPad Prism Suite 8.4.3.

Docking and molecular dynamics (MD) simulations. For MD simulations of $a_{1 B} A R$, the crystallographic pose of $(+)$-cyclazosin shown in Fig. 2a on the left was used as the starting pose. For $\alpha_{2 C} \mathrm{AR}$ (PDB ID: $6 \mathrm{KUW}^{26}$ ), the initial poses of the non-crystallographic ligands yohimbine and corynanthine were generated using the docking function of ICM-Pro (Molsoft LLC). The standard docking procedure involved defining receptor atoms within $5 \AA$ of the co-crystallized ligand RS79948 as the binding site of interest, around which a $25 \times 25 \times 25 \AA$ box was established. Docking was performed while maintaining the receptor as rigid, except for corynanthine docking, where L128 $8^{3.29}$ needed to be flexible in order to allow corynanthine to dock into the pocket. Typically, only the most energetically favorable docking poses were analyzed with MD, but stacks of up to 80 possible poses were visually inspected as part of the docking optimization process. MD preparation and simulations were conducted using the Desmond MD simulation system $^{83}$, as previously described ${ }^{84}$. For MD simulations of $\alpha_{1 B} A R$, the DARPin D12 fusion was excised. For MD simulations of $\alpha_{2 C} A R$, the $P$. abysii glycogen synthase fusion was removed. Any loop residues that were missing from the starting structures were left out in the MD simulations. Imported protein-ligand docked structures were pre-processed and minimized with OPLS3e force fields ${ }^{85}$ using the protein preparation wizard tool. Using the system builder tool, a 1palmitoyl-2-oleoyl-sn-glycero-3-phosphocholine (POPC) bilayer was built around the receptor with an additional $10 \times 10 \times 10 \AA$ orthorhombic buffer. The simple point charge (SPC) water model was used. The $\alpha_{1 \mathrm{~B}} \mathrm{AR}$ structure was neutralized by $12 \mathrm{Cl}^{-}$ions and the $\alpha_{2 \mathrm{C}} \mathrm{AR}$ structure by $13 \mathrm{Cl}^{-}$ions, and $\mathrm{Na}^{+}$and $\mathrm{Cl}^{-}$ions corresponding to $0.15 \mathrm{M} \mathrm{NaCl}$ were added to the system. Protein structures were first relaxed through a series of MD simulations. Initially, Brownian Dynamics was run at $10 \mathrm{~K}$ with restraints on solute heavy atoms for 100 ps under NVT conditions. This was followed by two simulations at $50 \mathrm{~K}$ with the protein and the Z-plane of the membrane restrained, first under NPT then NP $\gamma \mathrm{T}$ conditions. The next simulation involved gradual heating from 100 to $300 \mathrm{~K}$ with a gradual release of restraints, followed by a final relaxation step where restraints were removed. Production NP $\gamma$ T MD simulations were then run at $300.0 \mathrm{~K}$ and $1 \mathrm{~atm}$ for $300 \mathrm{~ns}$ with a recording interval of $300 \mathrm{ps}$. The membrane surface tension was set to $4000 \mathrm{bar} / \AA$. MD data were gathered using VMD 1.9.3 $3^{86}$. Protein and ligand root-mean-square deviation (RMSD) values were obtained using the RMSD visualizer tool. RMSD of ligands and protein backbone residues are relative to the receptor in its initial frame after the MD relaxation protocol described above.

Reporting summary. Further information on research design is available in the Nature Research Reporting Summary linked to this article.

\section{Data availability}

Coordinates and structure factors for the complex of $\alpha_{1 \mathrm{~B}} \mathrm{AR}_{\mathrm{XTAL}}$ and $(+)$-cyclazosin have been deposited in the worldwide PDB under the accession code: 7B6W. All data needed to evaluate the conclusions of the paper are present in the main manuscript and/or in the Supplementary Information. Additional data supporting the findings of this paper are available from the corresponding authors upon reasonable request. Additional publicly available PDB entries mentioned in this paper: 6KUW; 6KUX; 6KUY; 6K41; $2 \mathrm{YCW}$; 2RH1; 4LDO; 6IBL; 5LW2. Source data are provided with this paper. 
Received: 9 July 2021; Accepted: 21 December 2021;

Published online: 19 January 2022

\section{References}

1. Bylund, D. B. et al. International union of pharmacology nomenclature of adrenoceptors. Pharmacol. Rev. 46, 121-136 (1994).

2. Hieble, J. P. et al. International union of pharmacology. X. recommendation for nomenclature of $\alpha_{1}$-adrenoceptors: consensus update. Pharmacol. Rev. 47, 267-270 (1995).

3. Pupo, A. S. \& Minneman, K. P. Adrenergic pharmacology: focus on the central nervous system. CNS Spectr. 6, 656-662 (2001).

4. Finch, A. M. et al. In The Adrenergic Receptors: In The 21st Century (ed. Perez, D. M.) 25-147 (Humana Press, 2005).

5. Fuentes, A. V., Pineda, M. D. \& Venkata, K. C. N. Comprehension of top 200 prescribed drugs in the US as a resource for pharmacy teaching, training and practice. Pharmacy 6, 43 (2018).

6. Philipp, M., Brede, M. \& Hein, L. Physiological significance of $\alpha_{2}$-adrenergic receptor subtype diversity: one receptor is not enough. Am. J. Physiol. Regul. Integr. Comp. Physiol. 283, R287-R295 (2002).

7. Perez, D. M. \& Doze, V. A. Cardiac and neuroprotection regulated by $a_{1^{-}}$ adrenergic receptor subtypes. J. Recept. Signal Transduct. Res. 31, 98-110 (2011).

8. Akinaga, J., García-Sáinz, J. A. \& Pupo, A. S. Updates in the function and regulation of $\alpha_{1}$-adrenoceptors. Br. J. Pharmacol. 176, 2343-2357 (2019).

9. Perez, D. M. $\alpha_{1}$-adrenergic receptors in neurotransmission, synaptic plasticity, and cognition. Front. Pharmacol. 11, 1563 (2020).

10. Grisanti, L. A., Perez, D. M. \& Porter, J. E. Modulation of immune cell function by $\alpha_{1}$-adrenergic receptor activation. Curr. Top. Membr. 67, 113-138 (2011).

11. Staedtke, V. et al. Disruption of a self-amplifying catecholamine loop reduces cytokine release syndrome. Nature 564, 273-277 (2018).

12. Konig, M. F. et al. Preventing cytokine storm syndrome in COVID-19 using a-1 adrenergic receptor antagonists. J. Clin. Invest. 130, 3345-3347 (2020).

13. Rose, L. et al. The association between alpha-1 adrenergic receptor antagonists and in-hospital mortality from COVID-19. Front. Med. 8, 637647 (2021)

14. Philipp, M. et al. Placental $\alpha_{2}$-adrenoceptors control vascular development at the interface between mother and embryo. Nat. Genet. 31, 311-315 (2002).

15. Docherty, J. R. The pharmacology of $\alpha_{1}$-adrenoceptor subtypes. Eur. J. Pharmacol. 855, 305-320 (2019).

16. Michino, M. et al. What can crystal structures of aminergic receptors tell us about designing subtype-selective ligands? Pharmacol. Rev. 67, 198-213 (2015).

17. Kooistra, A. J., Kuhne, S., de Esch, I. J., Leurs, R. \& de Graaf, C. A structural chemogenomics analysis of aminergic GPCRs: lessons for histamine receptor ligand design. Br. J. Pharmacol. 170, 101-126 (2013).

18. Proudman, R. G. W., Pupo, A. S. \& Baker, J. G. The affinity and selectivity of $\alpha$-adrenoceptor antagonists, antidepressants, and antipsychotics for the human $\alpha 1 \mathrm{~A}, \alpha 1 \mathrm{~B}$, and $\mathrm{a1D}$-adrenoceptors. Pharmacol. Res. Perspect. 8, e00602 (2020).

19. Wang, $\mathrm{S}$. et al. $\mathrm{D}_{4}$ dopamine receptor high-resolution structures enable the discovery of selective agonists. Science 358, 381-386 (2017).

20. Wang, S. et al. Structure of the D2 dopamine receptor bound to the atypical antipsychotic drug risperidone. Nature 555, 269-273 (2018).

21. Zhou, Y., Cao, C., He, L. L., Wang, X. P. \& Zhang, X. J. C. Crystal structure of dopamine receptor D4 bound to the subtype selective ligand, L745870. eLife 8, e48822 (2019).

22. Fan, L. et al. Haloperidol bound $\mathrm{D}_{2}$ dopamine receptor structure inspired the discovery of subtype selective ligands. Nat. Commun. 11, 1074 (2020).

23. Wacker, D. et al. Crystal structure of an LSD-bound human serotonin receptor. Cell 168, 377-389 (2017).

24. Thal, D. M. et al. Crystal structures of the M1 and M4 muscarinic acetylcholine receptors. Nature 531, 335-340 (2016).

25. Liu, H. T. et al. Structure-guided development of selective M3 muscarinic acetylcholine receptor antagonists. Proc. Natl Acad. Sci. USA 115 12046-12050 (2018)

26. Chen, X. Y. et al. Molecular mechanism for ligand recognition and subtype selectivity of $\alpha_{2 C}$ adrenergic receptor. Cell Rep. 29, 2936-2943 (2019).

27. Vass, M. et al. Aminergic GPCR-ligand interactions: a chemical and structural map of receptor mutation data. J. Med. Chem. 62, 3784-3839 (2019).

28. Cherezov, V. et al. High-resolution crystal structure of an engineered human $\beta_{2}$-adrenergic G protein-coupled receptor. Science 318, 1258-1265 (2007).

29. Rasmussen, S. G. et al. Crystal structure of the $\beta_{2}$ adrenergic receptor- $G_{s}$ protein complex. Nature 477, 549-555 (2011).

30. Ring, A. M. et al. Adrenaline-activated structure of $\beta_{2}$-adrenoceptor stabilized by an engineered nanobody. Nature 502, 575-579 (2013).

31. Warne, T. et al. Structure of a $\beta_{1}$-adrenergic G-protein-coupled receptor. Nature 454, 486-491 (2008).
32. Moukhametzianov, R. et al. Two distinct conformations of helix 6 observed in antagonist-bound structures of a $\beta_{1}$-adrenergic receptor. Proc. Natl Acad. Sci. USA 108, 8228-8232 (2011).

33. Warne, T., Edwards, P. C., Doré, A. S., Leslie, A. G. W. \& Tate, C. G. Molecular basis for high-affinity agonist binding in GPCRs. Science 364, 775-778 (2019).

34. $\mathrm{Xu}, \mathrm{X}$. Y. et al. Binding pathway determines norepinephrine selectivity for the human $\beta_{1}$ AR over $\beta_{2}$ AR. Cell Res. 31, 569-579 (2020).

35. Qu, L. et al. Structural basis of the diversity of adrenergic receptors. Cell Rep. 29, 2929-2935 (2019)

36. Yuan, D. et al. Activation of the $\alpha_{2 B}$ adrenoceptor by the sedative sympatholytic dexmedetomidine. Nat. Chem. Biol. 16, 507-512 (2020)

37. Giardinà, D. et al. Synthesis and biological profile of the enantiomers of [4-(4amino-6,7-dimethoxyquinazolin-2-yl)-cis-octahydroquinoxalin-1-yl] furan-2ylmethanone (cyclazosin), a potent competitive alB-adrenoceptor antagonist. J. Med. Chem. 39, 4602-4607 (1996).

38. Giardinà, D. et al. Absolute configuration of the $\alpha_{1 \mathrm{~B}}$-adrenoceptor antagonist (+)-cyclazosin. Il Farm. 59, 965-969 (2004).

39. Rossier, O., Abuin, L., Fanelli, F., Leonardi, A. \& Cotecchia, S. Inverse agonism

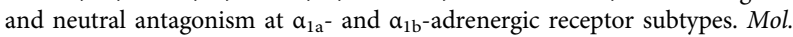
Pharmacol. 56, 858-866 (1999).

40. Ballesteros, J. A. \& Weinstein, H. Integrated methods for the construction of three-dimensional models and computational probing of structure-function relations in G protein-coupled receptors. In Methods in Neurosciences (ed. Sealfon, S. C.). Vol. 25, 366-428 (Academic Press, 1995).

41. Yong, K. J. et al. Determinants of ligand subtype-selectivity at $\alpha_{1 A^{-}}$ adrenoceptor revealed using saturation transfer difference (STD) NMR. ACS Chem. Biol. 13, 1090-1102 (2018).

42. Scott, D. J. \& Plückthun, A. Direct molecular evolution of detergent-stable G protein-coupled receptors using polymer encapsulated cells. J. Mol. Biol. 425, 662-677 (2013)

43. Mittl, P. R., Ernst, P. \& Plückthun, A. Chaperone-assisted structure elucidation with DARPins. Curr. Opin. Struct. Biol. 60, 93-100 (2020).

44. Deluigi, M. et al. Complexes of the neurotensin receptor 1 with smallmolecule ligands reveal structural determinants of full, partial, and inverse agonism. Sci. Adv. 7, eabe5504 (2021)

45. Alexandrov, A. I., Mileni, M., Chien, E. Y., Hanson, M. A. \& Stevens, R. C. Microscale fluorescent thermal stability assay for membrane proteins. Structure 16, 351-359 (2008).

46. Tickle, I. J. et al. STARANISO. http://staraniso.globalphasing.org (2018).

47. Ibrahim, P., Wifling, D. \& Clark, T. Universal activation index for class A GPCRs. J. Chem. Inf. Model. 59, 3938-3945 (2019).

48. Campbell, A. P., MacDougall, I. J., Griffith, R. \& Finch, A. M. An aspartate in the second extracellular loop of the $\alpha_{1 \mathrm{~B}}$ adrenoceptor regulates agonist binding. Eur. J. Pharmacol. 733, 90-96 (2014)

49. Campbell, S. F., Davey, M. J., Hardstone, J. D., Lewis, B. N. \& Palmer, M. J. 2,4-diamino-6,7-dimethoxyquinazolines. 1. 2-[4-(1,4-benzodioxan-2ylcarbonyl)piperazin-1-yl] derivatives as a1-adrenoceptor antagonists and antihypertensive agents. J. Med. Chem. 30, 49-57 (1987).

50. Bordner, J., Campbell, S. F., Palmer, M. J. \& Tute, M. S. 1,3-diamino-6,7dimethoxyisoquinoline derivatives as potential $\alpha_{1}$-adrenoceptor antagonists. J. Med. Chem. 31, 1036-1039 (1988).

51. Matijssen, C., Kinsella, G. K., Watson, G. W. \& Rozas, I. Computational study of the proton affinity and basicity of structurally diverse $\alpha_{1}$-adrenoceptor ligands. J. Phys. Org. Chem. 25, 351-360 (2012).

52. Milligan, C. M. et al. $\left[{ }^{3} \mathrm{H}\right]-\mathrm{RS}-79948-197$, a high affinity radioligand selective for $\alpha_{2}$-adrenoceptor subtypes. Ann. N. Y. Acad. Sci. 812, 176-177 (1997).

53. Uhlén, S. et al. $\left[{ }^{3} \mathrm{H}\right] \mathrm{RS} 79948-197$ binding to human, rat, guinea pig and pig $\alpha_{2 \mathrm{~A}^{-}}, \alpha_{2 \mathrm{~B}^{-}}$and $\alpha_{2 \mathrm{C}^{-}}$adrenoceptors. Comparison with MK912, RX821002, rauwolscine and yohimbine. Eur. J. Pharmacol. 343, 93-101 (1998).

54. Blaxall, H. S., Murphy, T. J., Baker, J. C., Ray, C. \& Bylund, D. B. Characterization of the alpha-2C adrenergic receptor subtype in the opossum kidney and in the OK cell line. J. Pharmacol. Exp. Ther. 259, 323-329 (1991).

55. Weinshank, R. L. et al. Cloning, expression, and pharmacological characterization of a human $\mathrm{a}_{2 \mathrm{~B}}$-adrenergic receptor. Mol. Pharmacol. 38 681-688 (1990).

56. Ruffolo, R. R., Bondinell, W. \& Hieble, J. P. $\alpha$ - and $\beta$-adrenoceptors: from the gene to the clinic. 2. Structure-activity relationships and therapeutic applications. J. Med. Chem. 38, 3681-3716 (1995).

57. Giardinà, D., Crucianelli, M., Melchiorre, C., Taddei, C. \& Testa, R. Receptor binding profile of cyclazosin, a new $\alpha_{1 \mathrm{~B}}$-adrenoceptor antagonist. Eur. J. Pharmacol. 287, 13-16 (1995).

58. Piscitelli, C. L., Kean, J., de Graaf, C. \& Deupi, X. A molecular pharmacologist's guide to G protein-coupled receptor crystallography. Mol. Pharmacol. 88, 536-551 (2015). 
59. Schuster, M. et al. Optimizing the $\alpha_{1 B}$-adrenergic receptor for solution NMR studies. Biochim. Biophys. Acta Biomembr. 1862, 183354 (2020).

60. Suryanarayana, S., Daunt, D. A., Vonzastrow, M. \& Kobilka, B. K. A point mutation in the seventh hydrophobic domain of the $\alpha_{2}$ adrenergic receptor increases its affinity for a family of $\beta$ receptor antagonists. J. Biol. Chem. 266, 15488-15492 (1991).

61. Simpson, M. M. et al. Dopamine D4/D2 receptor selectivity is determined by a divergent aromatic microdomain contained within the second, third, and seventh membrane-spanning segments. Mol. Pharmacol. 56, 1116-1126 (1999).

62. Gregory, K. J., Hall, N. E., Tobin, A. B., Sexton, P. M. \& Christopoulos, A. Identification of orthosteric and allosteric site mutations in $\mathrm{M}_{2}$ muscarinic acetylcholine receptors that contribute to ligand-selective signaling bias. $J$. Biol. Chem. 285, 7459-7474 (2010).

63. Leach, K., Davey, A. E., Felder, C. C., Sexton, P. M. \& Christopoulos, A. The role of transmembrane domain 3 in the actions of orthosteric, allosteric, and atypical agonists of the $\mathrm{M}_{4}$ muscarinic acetylcholine receptor. Mol. Pharmacol. 79, 855-865 (2011).

64. Keov, P. et al. Molecular mechanisms of bitopic ligand engagement with the $\mathrm{M}_{1}$ muscarinic acetylcholine receptor. J. Biol. Chem. 289, 23817-23837 (2014).

65. Abdul-Ridha, A. et al. Molecular determinants of allosteric modulation at the $\mathrm{M}_{1}$ muscarinic acetylcholine receptor. J. Biol. Chem. 289, 6067-6079 (2014).

66. Zhao, M. M., Hwa, J. \& Perez, D. M. Identification of critical extracellular loop residues involved in $\alpha_{1}$-adrenergic receptor subtype-selective antagonist binding. Mol. Pharmacol. 50, 1118-1126 (1996).

67. Sarkar, C. A. et al. Directed evolution of a G protein-coupled receptor for expression, stability, and binding selectivity. Proc. Natl Acad. Sci. USA 105, 14808-14813 (2008).

68. Wu, Y. F. et al. Rigidly connected multispecific artificial binders with adjustable geometries. Sci. Rep. 7, 11217 (2017).

69. Ernst, P. et al. Rigid fusions of designed helical repeat binding proteins efficiently protect a binding surface from crystal contacts. Sci. Rep. 9, 16162 (2019).

70. Leaver-Fay, A. et al. Rosetta3: an object-oriented software suite for the simulation and design of macromolecules. Methods Enzymol. 487, 545-574 (2011).

71. Egloff, P., Deluigi, M., Heine, P., Balada, S. \& Plückthun, A. A cleavable ligand column for the rapid isolation of large quantities of homogeneous and functional neurotensin receptor 1 variants from E. coli. Protein Expr. Purif. 108, 106-114 (2015).

72. Basu, S. et al. Automated data collection and real-time data analysis suite for serial synchrotron crystallography. J. Synchrotron Radiat. 26, 244-252 (2019).

73. Kabsch, W. Automatic processing of rotation diffraction data from crystals of initially unknown symmetry and cell constants. J. Appl. Crystallogr. 26, 795-800 (1993).

74. Evans, P. R. \& Murshudov, G. N. How good are my data and what is the resolution? Acta Crystallogr. D. Biol. Crystallogr. 69, 1204-1214 (2013).

75. Bailey, S. The CCP4 suite: programs for protein crystallography. Acta Crystallogr. D. Biol. Crystallogr. 50, 760-763 (1994).

76. McCoy, A. J. et al. Phaser crystallographic software. J. Appl. Crystallogr. 40, 658-674 (2007).

77. Emsley, P. \& Cowtan, K. Coot: model-building tools for molecular graphics. Acta Crystallogr. D. 60, 2126-2132 (2004).

78. Bricogne, G. et al. BUSTER v.2.10.3. http://www.globalphasing.com (2020).

79. Murshudov, G. N. et al. REFMAC5 for the refinement of macromolecular crystal structures. Acta Crystallogr. D. 67, 355-367 (2011).

80. Chen, V. B. et al. MolProbity: all-atom structure validation for macromolecular crystallography. Acta Crystallogr. D. 66, 12-21 (2010).

81. Cheng, Y. \& Prusoff, W. H. Relationship between the inhibition constant $\left(K_{\mathrm{i}}\right)$ and the concentration of inhibitor which causes 50 per cent inhibition $\left(I_{50}\right)$ of an enzymatic reaction. Biochem. Pharmacol. 22, 3099-3108 (1973).

82. Ehrenmann, J. et al. High-resolution crystal structure of parathyroid hormone 1 receptor in complex with a peptide agonist. Nat. Struct. Mol. Biol. 25, 1086-1092 (2018).

83. Bowers, K. J. et al. Scalable algorithms for molecular dynamics simulations on commodity clusters. in SC '06: Proceedings of the 2006 ACM/IEEE Conference on Supercomputing 43-43 (Tampa, 2006).

84. Vaid, T. M., Chalmers, D. K., Scott, D. J. \& Gooley, P. R. INPHARMA-based determination of ligand binding modes at $\alpha_{1}$-adrenergic receptors explains the molecular basis of subtype selectivity. Chem. Eur. J. 26, 11796-11805 (2020).

85. Roos, K. et al. OPLS3e: extending force field coverage for drug-like small molecules. J. Chem. Theory Comput. 15, 1863-1874 (2019).
86. Humphrey, W., Dalke, A. \& Schulten, K. VMD: visual molecular dynamics. J. Mol. Graph. Model. 14, 33-38 (1996).

\section{Acknowledgements}

We thank B. Blattmann and C. Müller-Simmen of the Protein Crystallization Center at the University of Zurich for their support during crystallization, and the staff of the X06SA beamline at the Paul Scherrer Institute for support during X-ray data collection. We thank G. Liechti-Tonarque and D. Barret for their help with protein expression, purification, and characterization, and C. Manatschal for support during the initial phase of refinement of the crystal structure. We thank M. Hilge for data processing and refinement of the crystal structure, and G. Murshudov for his help in the creation of the cyclazosin dictionary. We also would like to acknowledge V. Jameson of the Melbourne Cytometry Platform for assistance with FACS. This work was supported by Schweizerische Nationalfonds grant 31003A_182334 (to A.P.) and Australian National Health and Medical Research Council grants 1081801 and 1137179 (to D.J.S.).

\section{Author contributions}

D.J.S. and R.R.C. carried out directed evolution on $\alpha_{1 \mathrm{~B}} \mathrm{AR}$ and characterized single clones. M.D. designed the $\alpha_{1 \mathrm{~B}}$ AR-DARPin D12 fusion and the crystallization construct $\alpha_{1 B} A_{X T A L}$. M.D., L. Morstein., and L. Merklinger cloned, expressed, purified, crystallized $\alpha_{1 \mathrm{~B}} \mathrm{AR} \mathrm{R}_{\mathrm{XTL}}$, and harvested crystals. M.D. and M.S. established expression and purification protocols with support from O.Z., S.V., and P. E. M.D., L. Morstein, and A.K collected X-ray diffraction data. M.D. supervised S.V., L. Morstein, L. Merklinger, and A.K. in the aforementioned tasks, organized and planned the experiments. P.R.E.M. contributed to X-ray data processing and refinement in the initial phase of this project. M.D. analyzed and interpreted structural data. L. Morstein cloned receptor mutants, performed ligand-binding and signaling experiments, and analyzed the data with support from C.K. and M.D. S.A.E. supported L. Morstein in the initial phase of this project. D.J.S., L.A.d.Z., D.K.C., and T.M.V. performed docking and molecular dynamics simulations. Project management was carried out by M.D. and A.P. with support from D.J.S and O.Z. The manuscript was prepared by M.D., L. Morstein, D.J.S., and A.P. with inputs from all authors. All authors contributed to the final editing and approval of the manuscript.

\section{Competing interests}

The authors declare no competing interests.

\section{Additional information}

Supplementary information The online version contains supplementary material available at https://doi.org/10.1038/s41467-021-27911-3.

Correspondence and requests for materials should be addressed to Daniel J. Scott or Andreas Plückthun.

Peer review information Nature Communications thanks Arun Shukla and the other, anonymous, reviewer(s) for their contribution to the peer review of this work. Peer reviewer reports are available.

Reprints and permission information is available at http://www.nature.com/reprints

Publisher's note Springer Nature remains neutral with regard to jurisdictional claims in published maps and institutional affiliations.

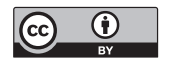

Open Access This article is licensed under a Creative Commons Attribution 4.0 International License, which permits use, sharing, adaptation, distribution and reproduction in any medium or format, as long as you give appropriate credit to the original author(s) and the source, provide a link to the Creative Commons license, and indicate if changes were made. The images or other third party material in this article are included in the article's Creative Commons license, unless indicated otherwise in a credit line to the material. If material is not included in the article's Creative Commons license and your intended use is not permitted by statutory regulation or exceeds the permitted use, you will need to obtain permission directly from the copyright holder. To view a copy of this license, visit http://creativecommons.org/ licenses/by/4.0/.

(C) The Author(s) 2022 